\title{
Formation of ice lenses and frost heave
}

\author{
A. W. Rempel ${ }^{1}$ \\ Received 13 April 2006; revised 26 January 2007; accepted 8 March 2007; published 21 June 2007.
}

[1] I examine the morphology of ice growth in porous media. Intermolecular forces cause premelted fluid to migrate and supply segregated ice growth (e.g., lenses) and frost heave. I account for the net effect of these microscopic interactions in a homogenized model formulated in terms of fundamental physical properties and characteristics of the porous medium that can be measured; no ad hoc parameterizations are required. Force equilibrium constraints yield the rate of fluid migration toward the ice lens boundary and predict the conditions under which new lenses are initiated. By combining this analysis with considerations of the heat flow problem in a step-freezing (Stefan) configuration, I elucidate the boundaries between different regimes of freezing behavior. At higher overburden pressures and relatively warm surface temperatures, ice lenses cannot form, and freezing of the available liquid occurs within the pore space, with no accompanying deformation. When conditions allow a lens to form, water is drawn toward it. If the fluid supply is sufficiently rapid, the lens grows faster than the latent heat of fusion can be carried away, and its boundary temperature warms until it reaches a stable steady state configuration. At lower fluid supply rates, the lens boundary temperature cools until a new lens can form at a warmer temperature beneath. With subsequent freezing this lens grows until yet another lens forms and the process repeats. An approximate treatment leads to estimates of the evolving lens thickness and spacing, as well as the accumulated total heave.

Citation: Rempel, A. W. (2007), Formation of ice lenses and frost heave, J. Geophys. Res., 112, F02S21, doi:10.1029/2006JF000525.

\section{Introduction}

[2] When water is frozen within porous media, interactions between the ice and matrix surfaces produce a diverse array of behavior, including the growth of needle ice (also known as pipkrake) at the ground surface, periodic lensing and heave in sediments, fracture of intact rock, and incorporation of sediment bands into the basal reaches of glaciers. These phenomena are promoted by the influence of mineral surfaces on the phase behavior of ice. A large body of experimental and computational (e.g., molecular dynamics) research confirms theoretical predictions that premelted liquid films coat ice surfaces in equilibrium at temperatures that are below the bulk melting temperature $\left(0^{\circ} \mathrm{C}\right.$ at atmospheric pressure) [see, e.g., Dash et al., 1995, 2006, and references therein]. These films provide fluid conduits that supply the growth of segregated ice even as the intermolecular forces responsible for their presence generate the substantial heaving pressures that cause frost damage.

[3] Models aimed at describing the dynamics of ice formation in porous media are complicated by the need to incorporate physical interactions that operate on the length

\footnotetext{
${ }^{1}$ Department of Geological Sciences, University of Oregon, Eugene, Oregon, USA.

Copyright 2007 by the American Geophysical Union. 0148-0227/07/2006JF000525\$09.00
}

scale of the premelted films, which are dwarfed by typical grain sizes yet control the macroscopic phenomena. Two primary approaches have been taken. In the first of these, attempts are made to account explicitly for the net interactions between the ice and mineral surfaces, with the latter treated using highly idealized geometries [e.g., Gilpin, 1980]. In part to circumvent the computational difficulties resulting from such direct calculations, the more common tact has been to introduce plausible, but nevertheless ad hoc parameterizations for the ice particle interactions, the choice of functional form being constrained to some extent by the empirical macroscopic behavior [e.g., Fowler and Krantz, 1994; Konrad and Morgenstern, 1981; O’Neill and Miller, 1985]. This second strategy, in particular, has enjoyed considerable success at elucidating the characteristics of ice growth under different environmental conditions. However, the force parameterizations frequently tend to obscure the underlying physics; the driving mechanisms have often been mistakenly attributed to more passive effects, such as pressure melting and the Gibbs-Thomson effect. Moreover, the absence of a firm connection between the microphysical processes and their macroscopic description produces considerable uncertainty in choosing how best to modify the model parameters when changing focus from one physical environment to the next.

[4] A modern understanding of premelting dynamics now makes it straightforward to formulate rigorous and computationally efficient treatments for the superposition of inter- 
molecular forces that drive segregated ice growth in natural porous media [Rempel et al., 2001]. These advances have been used recently to examine the freezing conditions within a simplified model that is focused on the dynamics of solidification under an imposed, steady thermal field [Rempel et al., 2004]. Here, I extend this work to predict how different freezing regimes develop within a transient thermal field, in particular for the case of a step-freezing configuration in a saturated, noncohesive soil. I derive a set of governing equations, based on conservation laws, to predict how the imposed environmental conditions produce the rich variety of behavior that can be observed within a single soil. The mechanical conditions that prevail within partially frozen porous media constrain the style of freezing that develops, whether it be the multiple lenses that are responsible for most of the frost damage that occurs within soils, the single-lens regime of needle ice, or the simple pore freezing that can occur without accompanying deformation. I detail these force equilibrium conditions in the next section, paying particular attention to the interplay between environmental conditions and the sediment characteristics that govern how the system responds. The energy balance in a Stefan, or step-freezing, configuration is examined in section 3. By considering the heat flow and mechanical conditions together I produce regime diagrams that delineate the type of freezing behavior expected for given combinations of overburden and surface temperature. Focusing on the multiple lensing regime, I propose approximate expressions for the evolution of lens spacing and thickness over time. The implications of these results and possible extensions to this work are discussed further in section 4, before offering a few concluding and summary remarks.

\section{Force Equilibrium in Partially Frozen Porous Media}

[5] The process of freezing and melting in natural porous media is sufficiently gradual that inertia is negligible and the forces are balanced. To focus on the essential mechanisms of ice segregation and frost damage, I assume that the pore space, with volume fraction $\phi$, is completely filled with a combination of liquid water and solid ice, and that no soluble impurities or gaseous phases are present. The ice saturation $S_{S}$ is the volume fraction of the pore space occupied by ice. Even in the absence of soluble impurities, liquid water remains in equilibrium with ice at subzero temperatures because of the combined effects of interfacial premelting and the Gibbs-Thomson effect [e.g., Cahn et al., 1992; Dash et al., 2006]. Additional liquid can persist in a metastable (supercooled) state within isolated pores because of the nucleation barrier to solidification. There is no nucleation barrier to melting [e.g., Oxtoby, 1999], so in general $S_{S}$ is dependent on the solidification history as well as the pore space geometry and the in situ temperature [e.g., Swainson and Schulson, 2001]. The effects of changes in pore pressure on $S_{S}$ are assumed negligible. I am primarily concerned with examining the system response to monotonic cooling so $S_{S}$ is treated as a known function of temperature $T$ alone. To develop intuition I focus on the case where the porous medium properties are laterally continuous and the temperature varies only in the vertical direction $z$, which is defined to increase with depth. I examine the force balance conditions beneath the lower surface of the warmest segregated lens, assuming that any pore ice beneath this level is attached and able to transmit forces through skeletal connections to the overlying ice [Miller, 1978] (see Figure 1).

\subsection{Forces Between Mineral Grains and the Ice}

[6] The most significant frost damage occurs when segregated ice grows and pushes apart mineral grains to produce macroscopic deformation of the porous medium. The ice growth is supplied through premelted liquid films that disjoin the ice from the mineral grains. It is the intermolecular interactions across these thin films that are the driving force for frost damage. The vector sum of the surface-normal interactions is the net thermomolecular force [Dash, 1989]. It has been shown that the net thermomolecular force acting on an inclusion surrounded by premelted ice (or an inclusion of premelted ice within a bounding substrate) is proportional to the mass of ice that could occupy the enclosed volume [Rempel et al., 2001], much as Archimedes' principle dictates that the net force on a floating object is proportional to the mass of displaced fluid. This result is used to calculate the net thermomolecular force exerted between the ice and the mineral grains that are found beneath a particular level within the partially frozen region.

[7] It is worth a brief digression to make note of a persistent misunderstanding in the frost heave literature concerning the origin of the force that drives heave. By analogy with the surface tension effects that lead to capillary rise in the vadose zone at temperatures above melting, it has often been suggested that the surface energy of the iceliquid interface, which is responsible for the Gibbs-Thomson effect, leads to the water migration that supplies ice lens growth. I emphasize here that this has been proven wrong [Rempel et al., 2001]. Because premelted water wets particle surfaces, there are no contacts between the ice and particle surfaces; this is quite different from the case of capillary rise, which is driven by the net force produced by surface tension at the air-water-particle contacts. The GibbsThomson effect does play an important role in contributing toward determining the permeability of partially frozen regions to fluid flow, as well as in setting the depth to the furthest extent of pore ice. However, the ice particle interactions that cause interfacial premelting and produce the net thermomolecular force are responsible for setting up the fluid pressure gradients that cause unfrozen water to flow and supply the lens growth that drives heave.

[8] For a porous medium of a given geometry, it is convenient to define the temperature to which ice can extend as $T_{f}$, and the corresponding depth as $z_{f}$ (see Figure 2). These are determined primarily by the influence of surface energy (e.g., the Gibbs-Thomson effect) in limiting the invasion of ice through restrictions in the pore space as the bulk melting temperature is approached. I focus on the case where the porous medium is partially ice saturated beneath the warmest lens at temperature $T_{l}<T_{f}$ and position $z_{l}<z_{f}$ so that a frozen fringe is formed. Of prime importance is the net effect of intermolecular interactions that separate the mineral grains from the contorted ice surface. Two quantities are actually needed: first, the net thermomolecular force that pushes upward on the ice that is beneath any given 


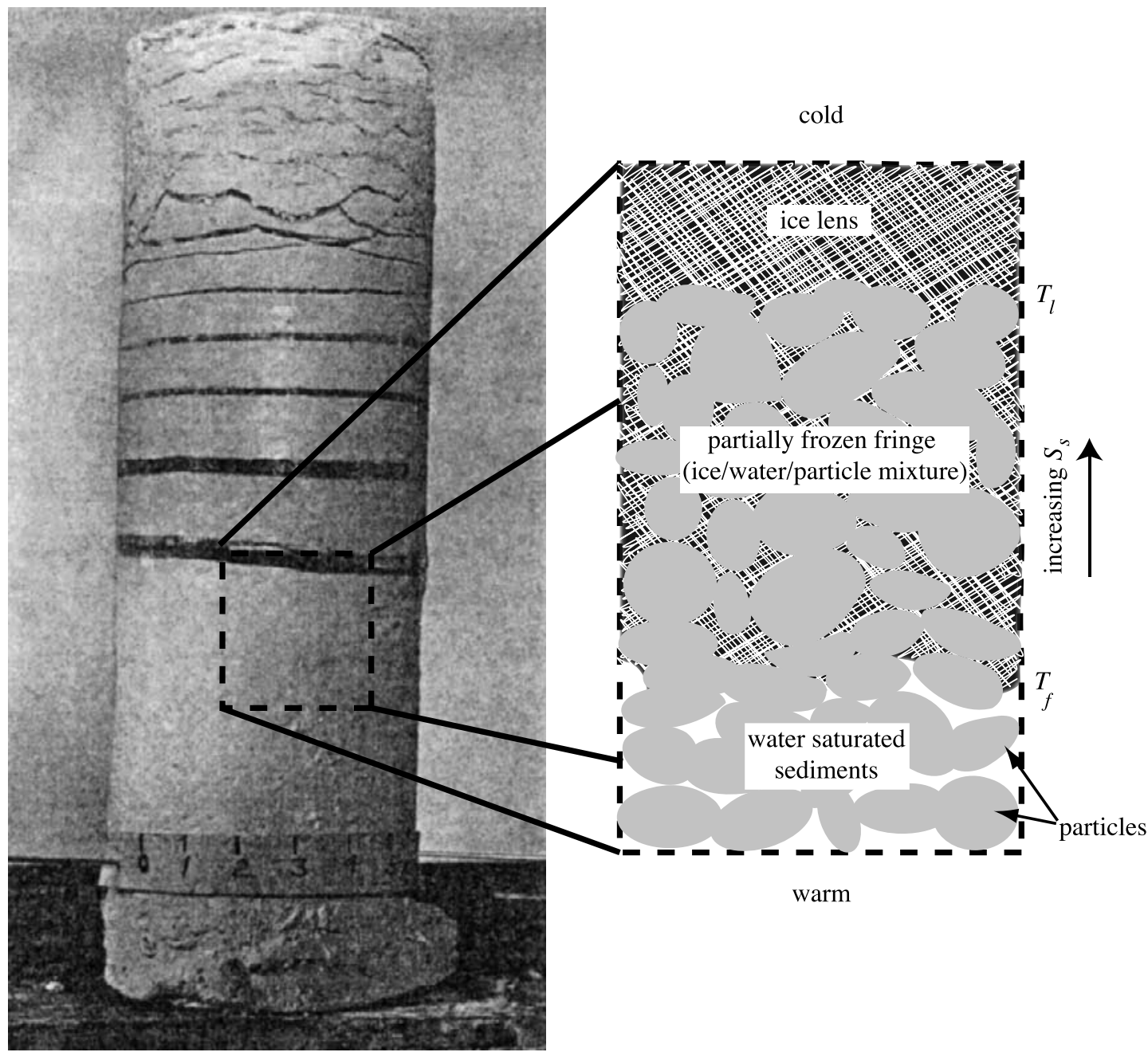

Figure 1. Photograph showing ice lenses (dark) that developed in a step-freezing experiment performed by Taber [1930], reprinted with permission of the University of Chicago Press. The schematic diagram shows the region of partial ice saturation that develops beneath the lowermost, growing ice lens.

isotherm within the frozen fringe; second, the net thermomolecular force that pushes upward on the lens itself, as a function of the temperature at its boundary. The following procedure homogenizes the microscopic interactions to provide a continuum description of these net forces.

[9] First, envision making a horizontal cut through the pore ice at some position $z$ within the frozen fringe. One can think of the newly disconnected ice beneath that level as being completely surrounded by a substrate, with an intervening premelted film. In Figure 2, the volume of ice between $z$ and the fringe base $\int_{z}^{z f} \phi S_{s} \mathrm{~d} z$ is represented by the stippled region beneath the dashed line. The principle of "thermodynamic buoyancy" outlined above implies that the net thermomolecular force per unit area on this ice body would be [Rempel et al., 2001]

$$
\mathbf{F}_{d}=\frac{\rho \mathcal{L}}{T_{m}} \int_{z}^{z_{f}} \phi S_{s} \nabla T \mathrm{~d} z=\frac{\rho \mathcal{L}}{T_{m}} \int_{T}^{T_{f}} \phi S_{S} \mathrm{~d} T \hat{\mathbf{z}},
$$

where $\rho \approx 920 \mathrm{~kg} / \mathrm{m}^{3}$ is the ice density, $\mathcal{L} \approx 3.3 \times 10^{5} \mathrm{~J} / \mathrm{kg}$ is the latent heat per unit mass, and $T_{m}(P)$ is the bulk melting temperature at the in situ pressure (e.g., $T_{m} \approx 273 \mathrm{~K}$ near the ground surface). The temperature gradient must be vertical for the second equality to hold. Now, since the ice is actually attached at its upper boundary, we subtract the force that would have been applied on that imaginary horizontal surface by the material just above $z$ (as shown in Figure 2, only a fraction $\phi S_{S}$ of the horizontal cross section at $z$ is occupied by ice). This gives us the net force per unit area exerted by the mineral grains on the ice beneath $z$ as

$$
\mathbf{F}_{T}(T)=-\frac{\rho \mathcal{L}}{T_{m}}\left[\left(\phi S_{s}\right)_{T=T^{-}}\left(T_{m}-T\right)-\int_{T}^{T_{f}} \phi S_{s} \mathrm{~d} T\right] \hat{\mathbf{z}},
$$

where $\hat{\mathbf{z}}$ is a unit vector. This is the vertical force that tends to separate mineral grains and leads to ice lens formation in porous media. The notation $\left(\phi S_{S}\right)_{T=T^{-}}$indicates that $\phi S_{S}$ should be evaluated immediately above the level $z$ (e.g., on the colder side). This distinction becomes important at the lens boundary, where the ice content $\phi S_{S}$ jumps discontinuously to unity. Hence equation (1) gives the net force per unit area over the entire lens boundary as

$$
\mathbf{F}_{T}\left(T_{l}\right)=-\frac{\rho \mathcal{L}}{T_{m}}\left(T_{m}-T_{l}-\int_{T_{l}}^{T_{f}} \phi S_{s} \mathrm{~d} T\right) \hat{\mathbf{z}} .
$$




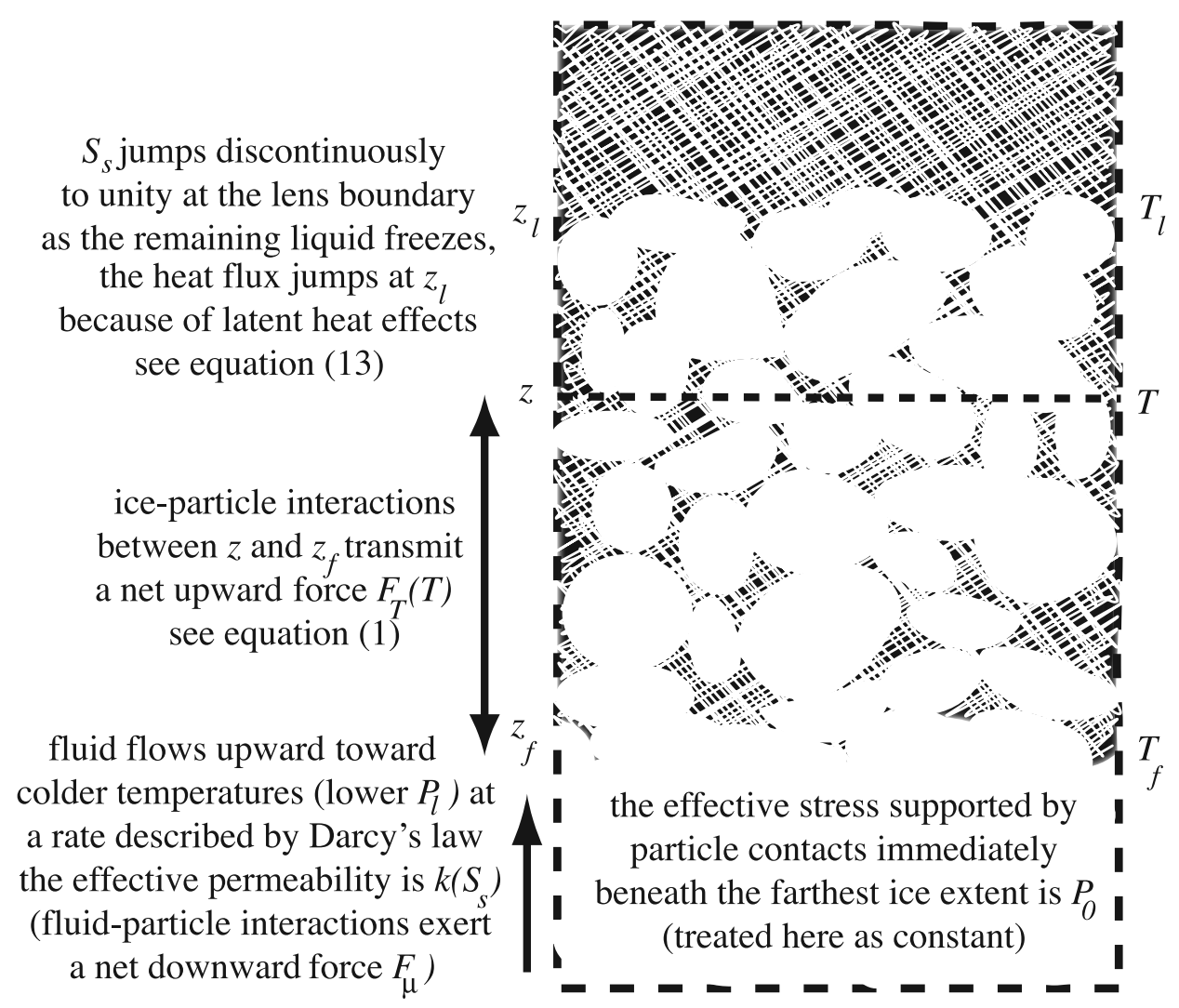

Figure 2. Schematic diagram of the region beneath a growing ice lens, showing several of the key interactions that are incorporated within the governing equations.

It may be shown that in the extension to cases where $T_{l}>T_{f}$, so that there is no ice beneath the lens, $\mathbf{F}_{T}\left(T_{l}\right)=$ $-\rho \mathcal{L}\left(T_{m}-T_{l}\right) / T_{m} \hat{\mathbf{z}}$.

[10] An important implication of equations (1) and (2) is that the net thermomolecular force per unit area does not depend directly on the details of the temperature profile. When the temperature dependence of the saturation profile is known, both the net thermomolecular force on the lowermost lens and the profile of net force on the connected pore ice beneath it are readily calculated as a function of temperature.

\subsection{Effective Overburden and Forces That Result From Fluid Flow}

[11] The overburden is defined so that $P_{0}=\left|\mathbf{F}_{T}\left(T_{l}\right)\right|$ when the distribution of fluid pressure on the lens boundary is hydrostatic. $P_{0}$ may be regarded as the effective stress at the furthest extent of connected ice, e.g., on the $T_{f}$ isotherm (or the $T_{l}$ isotherm when no fringe is present); $P_{0}$ is the force per unit area supported by particle contacts immediately beneath the ice.

[12] In the modeling that follows I treat the case of a constant $P_{0}$, as is appropriate when changes to the overburden are much slower than the dynamics of lens initiation and growth. For example, the experimental result shown in Figure 1 was obtained with an added heavy weight (not shown) on top of the sediment cylinder. However, one can easily envision circumstances where the overburden is more accurately represented as a function of lens position, for example when the surface load and the depth to the lens boundary are both small. In cases where the fluid supply through the unfrozen sediments imposes large hydrodynamic gradients that influence the fluid pressure on the $T_{f}$ isotherm, $P_{0}$ can be strongly dependent on the rate of lens growth. I ignore these complications for now to better focus on the essential mechanical considerations that determine the freezing morphology under the most elementary conditions.

[13] In general, $P_{0}$ and $\left|\mathbf{F}_{T}\left(T_{l}\right)\right|$ will not be exactly balanced; indeed, they are controlled by quite different phenomena, the former by gravity and the latter by heat flow. To satisfy Newton's first law, any mismatch must be compensated by the distribution of fluid pressure that acts against the ice boundary and produces a net hydrodynamic force per unit area $\mathbf{F}_{\mu}$ Because water flows much more easily than ice, for the purposes of calculating $\mathbf{F}_{\mu}$ the ice surface is regarded as rigid. It can support a shear stress. As in other problems in fluid dynamics that involve a thin film separated by two rigid surfaces [e.g., Batchelor, 1967, pp. 219-222], the liquid pressure at a given point cannot be determined by only considering the local forces on the bounding solids. Instead, an integral force balance condition must be satisfied.

[14] I assume that when a fringe is present, flow toward the lens boundary is adequately described by Darcy's law with an effective permeability $k$ that is a known function of the ice saturation level $S_{S}$ (or temperature $T<T_{f}$, since I assume ice saturation is a prescribed function of $T$ ). The hydrodynamic force per unit area acting on the ice and mineral grains beneath level $z$ can be written in terms of the 
surface integral of the liquid pressure $P_{l}$ over the ice-liquid interface. Using the divergence theorem, this is more usefully expressed as

$$
\begin{aligned}
\mathbf{F}_{\mu}(z) & =\int_{z}^{z_{f}}\left(\nabla P_{l}-\rho_{l} \mathbf{g}\right)\left(1-\phi S_{S}\right) \mathrm{d} z \\
& =-\int_{z}^{z_{f}} \frac{\mu}{k} \mathbf{U}\left(1-\phi S_{S}\right) \mathrm{d} z
\end{aligned}
$$

where the volume fraction of liquid in the fringe is $\left(1-\phi S_{s}\right)$, $\mu \approx 1.8 \times 10^{-3} \mathrm{~Pa} \mathrm{~s}$ is the liquid viscosity, and $\mathbf{U}$ is the Darcy flow velocity. When no fringe is present a similar expression arises, with the permeability reinterpreted to describe the resistance to flow in the films that separate the ice from the adjacent mineral grains beneath [Worster and Wettlaufer, 1999]. The continuity condition implies that $\rho_{l} \mathbf{U}+\rho\left(1-\phi S_{s}\right) \mathbf{V}_{l}=0$, where $\mathbf{V}_{l}$ is the rate of segregated ice growth: the heave rate (when $\mathbf{V}_{l}$ is oriented in the positive $\hat{z}$ direction, the ground surface is driven upward).

[15] It is useful to express all the forces in the system in terms of temperature rather than position. I assume that $S_{s}$ varies only with temperature and that the permeability $k$ is a function of $S_{s}$. Equation (3) can be written as

$$
\begin{aligned}
\mathbf{F}_{\mu} & =\mu \frac{\rho}{\rho_{l}} \mathbf{V}_{l} \int_{z}^{z_{f}} \frac{\left(1-\phi S_{s}\right)^{2}}{k} \mathrm{~d} z \\
& =\mu \frac{\rho}{\rho_{l}} \mathbf{V}_{l} \int_{T}^{T_{f}} \frac{\left(1-\phi S_{s}\right)^{2}}{k|\nabla T|} \mathrm{d} T,
\end{aligned}
$$

where I have used the continuity condition to write $\mathbf{F}_{\mu}$ in terms of the heave rate.

\subsection{Constraints on Lens Growth and Decay}

[16] Over the lens boundary, force equilibrium requires that the net thermomolecular force balance the effective overburden and the hydrodynamic forces that result from fluid flow. Accordingly, the above considerations lead to

$$
\begin{aligned}
P_{0} & +\mu \frac{\rho}{\rho_{l}}\left(\mathbf{V}_{l} \cdot \hat{\mathbf{z}}\right) \int_{T_{l}}^{T_{f}} \frac{\left(1-\phi S_{S}\right)^{2}}{k|\nabla T|} \mathrm{d} T \\
& -\frac{\rho \mathcal{L}}{T_{m}}\left(T_{m}-T_{l}-\int_{T_{l}}^{T_{f}} \phi S_{s} \mathrm{~d} T\right)=0,
\end{aligned}
$$

which describes the relationship between the overburden, the heave rate, the thermal field, and the lens temperature. The lens grows when equation (5) is satisfied with $\mathbf{V}_{l}$ in the $\hat{\mathbf{z}}$ direction, it melts when $P_{0}$ is sufficiently large that $\mathbf{V}_{l} \cdot \hat{\mathbf{z}}<0$.

[17] In circumstances where the temperature gradient through the fringe is nearly uniform, I can approximate $|\nabla T|$ by the average gradient $\left(T_{f}-T_{l}\right) /\left(z_{f}-z_{l}\right)$. Even when deviations from a linear temperature gradient are important, it is useful to write equation (5) as

$$
\left(\mathbf{V}_{l} \cdot \hat{\mathbf{z}}\right)\left(z_{f}-z_{l}\right)=H\left(T_{l}\right) \psi\left(z_{l}, T_{l}\right),
$$

where $\psi(z, T)$ will be defined momentarily, and the capacity for heaving

$$
H\left(T_{l}\right)=\left[\frac{\rho_{l} \mathcal{L}\left(T_{f}-T_{l}\right)}{\mu T_{m}}\right] \frac{T_{m}-T_{l}-\int_{T_{l}}^{T_{f}} \phi S_{s} \mathrm{~d} T-P_{0} T_{m} /(\rho \mathcal{L})}{\int_{T_{l}}^{T_{f}}\left[\left(1-\phi S_{s}\right)^{2} / k\right] \mathrm{d} T},
$$

with units of diffusivity, depends on pore space characteristics, the overburden, and material properties. With its dependence on the product of heave rate $\mathbf{V}_{l}$ and fringe thickness $z_{f}-z_{l}, H$ can be thought of as describing the diffusion of water mass toward the ground surface; it is a measure of the contribution that the fluid pressure distribution makes toward the force balance on the lens. $H$ is large when the force balance over the lens boundary causes a reduction in the fluid pressure that allows water to be drawn relatively quickly through the frozen fringe to supply lens growth (e.g., high $\mathbf{V}_{l}$ ); when the fringe is thicker (e.g., high $z_{f}-z_{l}$ ), the rate of this fluid supply and the consequent lens growth is lower than it would be for a shorter path through the partially frozen sediments. The details of the temperature profile enter into equation (6) through the function

$$
\psi(z, T)=\left(\frac{z_{f}-z_{l}}{T_{f}-T_{l}}\right) \frac{\int_{T}^{T_{f}}\left[\left(1-\phi S_{s}\right)^{2} / k\right] \mathrm{d} T}{\int_{z}^{z_{f}}\left[\left(1-\phi S_{s}\right)^{2} / k\right] \mathrm{d} z},
$$

which is defined so that the upper integral takes place over temperature and the lower integral takes place over space, implying that $\psi\left(z_{l}, T_{l}\right) \equiv 1$ when the temperature gradient is uniform. When the temperature gradient changes appreciably through the fringe, the deviations of $\psi\left(z_{l}, T_{l}\right)$ from unity are expected to often be smaller than the uncertainty in $k\left(S_{S}\right)$, so that their neglect might be justified.

[18] The main advantage of breaking the right side of equation (6) into two separate terms is that it enables us to isolate the heaving capacity $H$, which is independent of the temperature profile, from a scaling factor $\psi$ of order unity that takes proper account of the comparatively minor variations in behavior that are produced by deviations from a uniform temperature gradient. Because the value of $H$ is the primary control on the rate of fluid mass transfer through the frozen fringe, it gauges the capacity for heave of a given porous system in a broad range of environmental conditions. I elaborate on this point momentarily, after first describing the mechanical constraint on lens initiation.

\subsection{Constraints on Lens Initiation}

[19] Beneath the lens boundary, force equilibrium requires that

$$
\begin{aligned}
P_{0} & +\mu \frac{\rho}{\rho_{l}}\left(\mathbf{V}_{l} \cdot \hat{\mathbf{z}}\right) \int_{T}^{T_{f}} \frac{\left(1-\phi S_{s}\right)^{2}}{k|\nabla T|} \mathrm{d} T \\
& -\frac{\rho \mathcal{L}}{T_{m}}\left[\phi S_{s}\left(T_{m}-T\right)-\int_{T}^{T_{f}} \phi S_{s} \mathrm{~d} T\right]=P_{p},
\end{aligned}
$$

where $P_{p}$ is the force per unit area that is borne by interparticle, or mineral, contacts. Note that the force balance condition immediately beneath the furthest extent of pore ice (or the lens boundary when the fringe is absent) simplifies to $P_{0}=P_{p}$. This emphasizes the connection between $P_{p}$ and effective stress. I assume that new lenses are initiated when $P_{p}=0$, as is appropriate for solidification in porous materials with negligible cohesion. In the discussion that follows I treat $\phi$ as constant. The appropriate extension to incorporate poroelastic effects would involve treating $\phi$ as a function of $P_{p}$. The use of empirical relations between $\phi$ and $P_{p}$, such as those derived from studies on the 


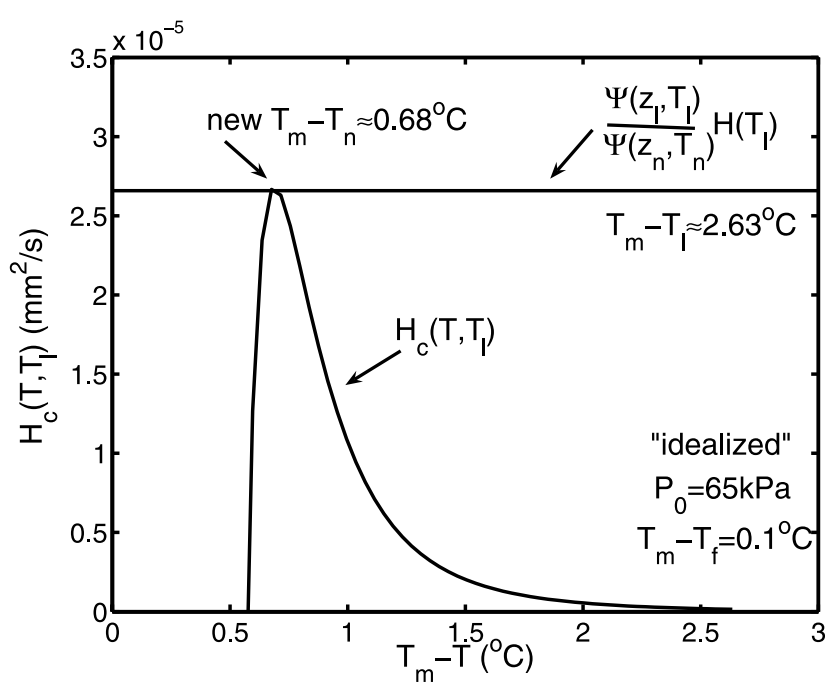

Figure 3. Diagnostic diagram for lens formation. The first lens forms where $H_{c}\left(T, T_{l}\right)=0$ at $T_{m}-T \approx 0.57^{\circ} \mathrm{C}$. Within the partially frozen fringe beneath the lens, the maximum value of $H_{c}\left(T, T_{l}\right)$ increases as the lens temperature drops. The profile shown here gives $H_{c}\left(T, T_{l}\right)$ evaluated for a lens temperature of $T_{l} \approx T_{m}-2.63^{\circ} \mathrm{C}$ and the parameters summarized in the first column of Table 1 . This is the lens temperature at which $H_{c}\left(T, T_{l}\right)$ first meets the horizontal line, which represents $H\left(T_{l}\right)$ scaled by the ratio $\psi\left(z_{l}, T_{l}\right) /$ $\psi\left(z_{n}, T_{n}\right) \approx 0.93$. At this intersection point, where the temperature is $T_{n} \approx T_{m}-0.68^{\circ} \mathrm{C}$, the load borne by particle contacts vanishes, and a new lens can form.

properties of glacial till above the melting transition [e.g., Clarke, 1987; Tulaczyk et al., 2000; Fowler, 2003], is the subject of ongoing experimental research (J. S. Wettlaufer, personal communication, 2005).

[20] Recalling the definitions for $H$ and $\psi$ given above, equation (7) implies that $P_{p}=0$ and new lenses can form when

$$
H_{c}\left(T, T_{l}\right)=\frac{\psi\left(z_{l}, T_{l}\right)}{\psi(z, T)} H\left(T_{l}\right),
$$

where the critical heaving capacity is defined for $T_{f}>T>T_{l}$ as

$H_{c}\left(T, T_{l}\right) \equiv\left[\frac{\rho_{l} \mathcal{L}\left(T_{f}-T_{l}\right)}{\mu T_{m}}\right] \frac{\left(T_{m}-T\right) \phi S_{s}-\int_{T}^{T_{f}} \phi S_{s} \mathrm{~d} T-P_{0} T_{m} /(\rho \mathcal{L})}{\int_{T}^{T_{f}}\left[\left(1-\phi S_{s}\right)^{2} / k\right] \mathrm{d} T}$

This gives a lens formation criterion that is only a function of temperature, overburden, and known material properties and pore space characteristics. Note that the ratio $\psi\left(z_{l}, T_{l}\right) /$ $\psi(z, T)=1$ when the temperature gradient through the fringe is constant and it approaches unity as $z$ approaches $z_{l}$. Equation (8) indicates that new lenses can form once the critical heaving capacity $H_{c}\left(T, T_{l}\right)$ at a level within the frozen fringe where the temperature is $T$ has increased enough to equal the scaled capacity for heaving $\left[\psi\left(z_{l}, T_{l}\right) /\right.$ $\psi(z, T)] H\left(T_{l}\right)$ at the old lens temperature $T_{l}$. This condition is attained when the fluid pressure distribution on the pore ice beneath the level $z$ exerts the same net force as the fluid pressure distribution over the entire lens boundary so that the particle contacts at $z$ no longer support any weight. Following a brief description of our choice of model parameters, I illustrate this balance in Figures 3 and 4.

\subsection{Parameter Values}

[21] Table 1 summarizes controlling parameter values for three different porous media, chosen to illustrate a range of potential solidification behavior. Relevant properties for the ice-water system are given in the upper portion of Table 1 . For each porous medium, identical values are chosen for the heat capacity, thermal diffusivity, and porosity. Appreciable changes to the thermal properties can occur as the ice saturation level changes, but I neglect these effects to better focus on the essential mechanical conditions that control the freezing morphology. Andersland and Ladanyi [2004, Table 2-6] tabulate parameters that describe the unfrozen water content as a function of temperature in a variety of different clays and silts. The manner in which the hydraulic conductivity depends on temperature is also reported for a few of these media [Andersland and Ladanyi, 2004, Figure C-14] (reproduced from Nixon [1991]). These empirical data indicate that the permeabilities and ice saturation levels can be written as

$$
\begin{gathered}
k(T)=k_{0}\left(\frac{T_{m}-T_{f}}{T_{m}-T}\right)^{\alpha} \\
S_{s}(T)=1-\left(\frac{T_{m}-T_{f}}{T_{m}-T}\right)^{\beta},
\end{gathered}
$$

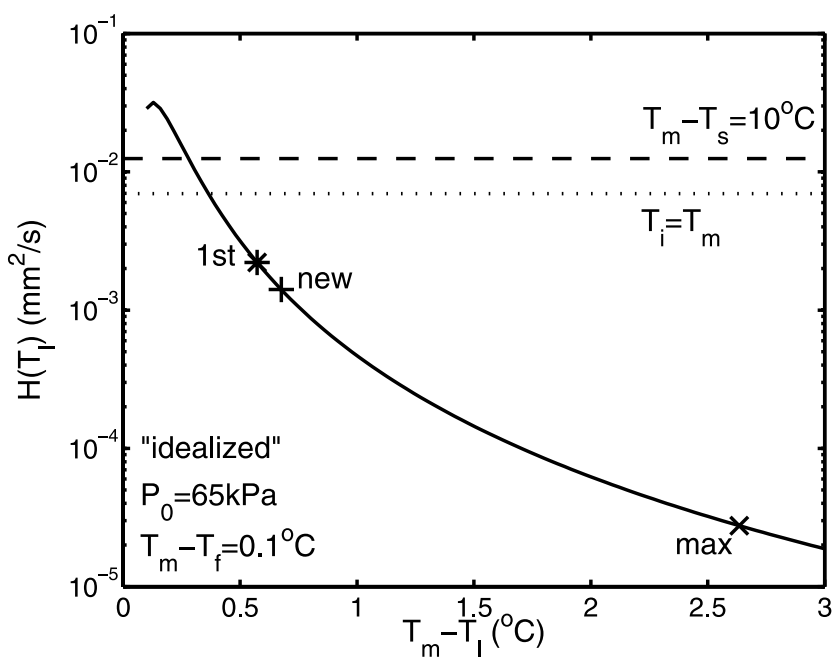

Figure 4. Plot of $H\left(T_{l}\right)$, which characterizes water diffusion during segregated ice growth. The first lens forms at $T_{m}-T_{l} \approx 0.57^{\circ} \mathrm{C}$, as indicated by the intercept in Figure 3 . When the rate of lens growth is lower than the rate of heat flow, the temperature at the lens boundary subsequently decreases, and the force balance is satisfied with the product of heave rate and fringe thickness equal to $H\left(T_{l}\right)$, scaled by $\psi$, which is of order unity. Once the lens temperature decreases to the point labeled "max" at $T_{m}$ $T_{l} \approx 2.63^{\circ} \mathrm{C}$, the effective stress borne by particle contacts disappears, and a new lens can form at the point labeled "new," where $T_{m}-T_{l} \approx 0.68^{\circ} \mathrm{C}$. The horizontal lines pertain to the calculation shown in Figure 9. 
Table 1. Parameters That Control Freezing Morphology ${ }^{a}$

\begin{tabular}{lccc}
\hline Parameter & Idealized & Chena Silt & Inuvik Clay \\
\hline$\rho, \mathrm{g} / \mathrm{cm}^{3}$ & 0.92 & 0.92 & 0.92 \\
$\rho_{l}, \mathrm{~g} / \mathrm{cm}^{3}$ & 1.00 & 1.00 & 1.00 \\
$\mathcal{L}, \mathrm{kJ} / \mathrm{kg}$ & 334 & 334 & 334 \\
$T_{m}, \mathrm{~K}$ & 273 & 273 & 273 \\
$\mu, \mathrm{mPa} \mathrm{s}$ & 1.8 & 1.8 & 1.8 \\
$\mathcal{C}, \mathrm{kJ} /\left(\mathrm{kg} \cdot{ }^{\circ} \mathrm{C}\right)$ & 1.3 & 1.3 & 1.3 \\
$\kappa, \mathrm{mm}^{2} / \mathrm{s}$ & 0.7 & 0.7 & 0.7 \\
$\phi$ & 0.35 & 0.35 & 0.35 \\
$T_{m}-T_{f},{ }^{\circ} \mathrm{C}$ & 0.1 & 0.031 & 2.81 \\
$\kappa_{0}, \mathrm{~m}^{2}$ & $10^{-15}$ & $4.1 \times 10^{-17}$ & $1.4 \times 10^{-19}$ \\
$\alpha$ & 4 & 3.1 & 1.4 \\
$\beta$ & 2 & 0.531 & 0.254 \\
\hline
\end{tabular}

${ }^{\text {a}}$ Properties of the ice water system and those common to all calculations that follow are shown in the top two thirds of the table, whereas the bottom third lists empirical parameters used in equations (9) and (10) to characterize $S_{s}$ and $k$ for each of three model porous media. The first column corresponds to an idealized case in which $S_{s}$ and $k$ are controlled by the presence of fluid channels with effective hydraulic radii determined by the Gibbs-Thomson effect. The second and third columns are derived from empirical data compiled by Andersland and Ladanyi [2004, Table 2-6 and Figure C-14] (originally from Nixon [1991]). For Chena silt, the data on which the permeability function is based derive from work by Horiguchi and Miller [1983]. Andersland and Ladanyi [2004, Table 2-6] reported Smith and Tice [1988] as the source of the saturation data, but the referenced paper does not appear to contain this information; it can be found instead in work by Tice et al. [1984]. For the Inuvik clay, both the saturation and permeability functions derive from the data of Smith [1985]

for $T<T_{f}$ (see Appendix A for further discussion). Theoretical considerations [e.g., Cahn et al., 1992] suggest the use of slightly more complicated empirical formulations that include at least one additional term in each of these expressions to account for variations in the thermal responses of different premelting mechanisms; however, equations (9) and (10) are sufficient for current purposes.

[22] The values of $T_{f}, \alpha$ and $\beta$ that characterize Chena silt and Inuvik clay are given in Table 1. Cahn et al. [1992] present a model for the effects of surface energy and interfacial premelting in controlling the ice saturation level in idealized packings of monodispersed particles. Experimental comparisons support the dominance of the GibbsThomson effect in controlling $S_{S}$ at temperatures that approach $T_{f}$, whereas interfacial premelting accounts for a larger fraction of the unfrozen water at colder temperatures. The first column in Table 1 gives permeability and ice saturation parameters that are consistent with the temperature dependence expected when the dominant water pathways have effective hydraulic radii controlled by surface-energy effects. I regard this as a limiting case in that the ice saturation, in particular, exhibits a much stronger temperature dependence for the idealized medium than for either of the other two porous media. The low values of $\beta$ for the Chena silt and Inuvik clay suggest that the liquid along premelted films occupies a much greater volume fraction than is contained within regions of high surface curvature. As discussed further by Cahn et al. [1992], the increased importance of liquid films at temperatures that are sufficiently removed from $T_{f}$ is expected to be a general characteristic of the freezing behavior of porous media; studies focused on the freezing behavior of silts and clays in the immediate vicinity of $T_{f}$ would be expected to yield higher values of $\beta$.

[23] One can gain intuition for the frost heaving behavior by neglecting variations in the fringe temperature gradient in order to evaluate approximate force balance conditions. As discussed above, this is equivalent to setting $\psi(z, T)=1$. More generally, even when latent heat effects cause the temperature gradient to undergo considerable spatial variations, the variations in $\psi$ tend to be much smaller than the changes that characterize the dependence of $H$ on temperature. For example, using the "idealized" porous media properties in Table $1, H$ changes by almost two orders of magnitude in a step-freezing configuration that sees $\psi$ change by less than a factor of two (e.g., see Figure 4 and Table 2). This gives us confidence that the insight gained into the dominant characteristics of soil freezing in a particular heat flow scenario provides a reasonable guide for evaluating the heaving behavior under other heat flow scenarios as well. Accordingly, I illustrate the heaving behavior of the model idealized soil for the step-freezing configuration that will be detailed further below.

[24] Figures 3 and 4 show the dependance of $H_{c}\left(T, T_{l}\right)$ on temperature and the dependence of $H\left(T_{l}\right)$ on lens temperature, evaluated for the idealized soil. (Appendix A provides analytical expressions for $H_{c}$ and the components of $H$ using the formulas for $k$ and $S_{s}$ given in equations (9) and (10).) An examination of equation (7) for the special case where $\mathbf{V}_{l} \equiv 0$ leads us to expect that the first lens will form at the temperature where $H_{c}\left(T, T_{l}\right)=0$; in this case $T_{m}-$ $T \approx 0.57^{\circ} \mathrm{C}$, as indicated by the $x$ intercept in Figure 3 , and shown with an asterisk on Figure 4 . This first lens begins growing so that $H\left(T_{l}\right) \approx 0.0022 \mathrm{~mm}^{2} / \mathrm{s}$, i.e., the product of the fringe thickness and the heave rate, divided by $\psi$. When

Table 2. Lens Temperatures Calculated for Three Different Types of Porous Media (With Properties Summarized in Table 1) at the Formation of the First Lens, Subsequent New Lenses, and Lenses at Their Maximum Extent ${ }^{\mathrm{a}}$

\begin{tabular}{lccc}
\hline \multicolumn{1}{c}{ Parameter } & Idealized & Chena Silt & Inuvik Clay \\
\hline$T_{m}-T_{f},{ }^{\circ} \mathrm{C}$ & 0.1 & 0.031 & 2.81 \\
$T_{m}-T_{s},{ }^{\circ} \mathrm{C}$ & 10 & 10 & 10 \\
$P_{0}, \mathrm{kPa}$ & 65 & 65 & 65 \\
$P_{0} / P_{\max }\left(T_{s}\right)$ & 0.83 & 0.34 & 0.11 \\
$T_{m}-T_{1 s t},{ }^{\circ} \mathrm{C}$ & 0.57 & 1.27 & 3.48 \\
$\lambda_{l 1}$ & 0.145 & 0.071 & 0.109 \\
$\lambda_{f 1}$ & 0.207 & 0.134 & 0.253 \\
$\psi\left(z_{l}, T_{l}\right)$ & 1.79 & 4.35 & 1.00 \\
$T_{m}-T_{n e w},{ }^{\circ} \mathrm{C}$ & 0.68 & 1.66 & $5.06{ }^{\mathrm{b}}$ \\
$\lambda_{l n}$ & 0.142 & 0.018 & 0.082 \\
$\lambda_{f n}$ & 0.157 & 0.118 & 0.121 \\
$\psi\left(z_{l}, T_{l}\right)$ & 1.03 & 4.66 & 1.00 \\
$T_{m}-T_{\max },{ }^{\circ} \mathrm{C}$ & 2.63 & 4.86 & $10^{\mathrm{b}}$ \\
$\lambda_{l m}$ & 0.113 & 0.010 & 0 \\
$\lambda_{f m}$ & 0.150 & 0.050 & 0.476 \\
$\psi\left(z_{l}, T_{l}\right)$ & 0.96 & 3.73 & 1.13 \\
\hline
\end{tabular}

${ }^{a}$ Also shown are the corresponding scaled lens coordinates and $\psi\left(z_{l}, T_{l}\right)$ from the approximate model discussed in section 3.3 (calculated with $T_{i}=$ $\left.T_{m}\right)$.

${ }^{b}$ For the Inuvik clay, if the surface temperature is sufficiently cold, the lens continues to grow until its temperature reaches $T_{m}-T_{\max } \approx 64.9^{\circ} \mathrm{C}$; only then are new lenses initiated at $T_{m}-T_{\text {new }} \approx 5.06^{\circ} \mathrm{C}$. When the surface temperature is warmer than $T_{\max }$, the lens temperature tends toward $T_{s}$ and $\lambda_{l} \rightarrow 0$ at large times. 
the heat flow is sufficiently rapid that the lens temperature subsequently decreases, Figure 4 indicates that $H\left(T_{l}\right)$ decreases until the point labeled with an ' $\mathrm{x}$ ' at $T_{m}-T_{l} \approx$ $2.63^{\circ} \mathrm{C}$ and $H\left(T_{l}\right) \approx 2.8 \times 10^{-5} \mathrm{~mm}^{2} / \mathrm{s}$. This is when the two solid lines in Figure 3 first intersect, indicating that the effective stress supported by particle contacts vanishes. A new lens is expected to form at this point, as labeled with a plus sign in Figure 3 at $T_{m}-T_{l} \approx 0.68^{\circ} \mathrm{C}$ where $H\left(T_{l}\right) \approx$ $0.0014 \mathrm{~mm}^{2} / \mathrm{s}$.

[25] In circumstances where heat flow is slow enough that the lens temperature increases, I would expect $H\left(T_{l}\right)$ to increase and the fringe to disappear once $T_{l}=T_{f}$, e.g., $T_{m}-$ $T_{l}=0.1{ }^{\circ} \mathrm{C}$ for the case shown. The calculation for Figure 4 did not extend to warmer temperatures, but a maximum in $H\left(T_{l}\right)$ is reached just before the fringe disappears for this choice of parameter values. When $H\left(T_{l}\right)$ is generalized appropriately to treat cases where the fringe is absent, $H\left(T_{l}\right)$ drops as the lens continues to warm. A steady state lens temperature can eventually be reached, the value of which depends on the imposed conditions.

\section{Stefan Problem}

[26] In addition to the mechanical conditions described above, the rate and morphology of ice growth is constrained by the conservation of energy. The temperature profile that results determines the deviation of $\psi(z, T)$ from unity. To give a specific example of the dynamics of periodic lens formation in a relatively simple configuration, I examine the Stefan problem [e.g., Wettlaufer, 2001] for ice growth in a uniform, noncohesive porous medium. Initially, the temperature $T(z, 0)=T_{i} \geq T_{f}$ throughout. The boundary temperature at $z=0$ is dropped at time $t=0^{+}$to $T(0, t)=$ $T_{s}<T_{f}$ and thereafter held constant. Three separate regions can develop: a lensed region between $z=0$ and $z_{l}(t)$, a partially frozen fringe between $z=z_{l}(t)$ and $z_{f}(t)$, and an ice-free region for $z>z_{f}(t)$. The fringe boundary temperature at position $z_{f}(t)$ is fixed at $T_{f}$ by the geometry of the pore space [Rempel et al., 2004]. The lens boundary position $z_{l}(t)$ and temperature $T_{l}(t)$ must be determined as part of the solution to the problem.

[27] To focus on the essential features of the problem, I ignore differences between the thermal properties of the system components (e.g., ice, water, and mineral particles), and I neglect the effects of density differences between the components on the heat capacity of the mixture. Considering only conductive heat transfer, with effective thermal diffusivity $\kappa$, the energy conservation requires that

$$
\frac{\partial T}{\partial t}=\kappa \frac{\partial^{2} T}{\partial z^{2}}
$$

both for $z_{l}(t)>z>0$ and for $z>z_{f}(t)$. In the fringe, where $z_{f}(t)>z>z_{l}(t)$, the consumption of latent heat and the net advection of pore ice that accompanies heave at rate $\mathbf{V}_{l}$ modify the thermal evolution so that [cf. O'Neill and Miller, 1985, equation (19)]

$$
\left(\frac{\partial T}{\partial t}+\mathbf{V}_{l} \frac{\partial T}{\partial z}\right)\left(1-\frac{\mathcal{L} \phi}{\mathcal{C}} \frac{\mathrm{d} S_{s}}{\mathrm{~d} T}\right)=\kappa \frac{\partial^{2} T}{\partial z^{2}}
$$

where $\mathcal{C}$ is heat capacity. Note that the ice saturation level decreases with temperature so that $\mathrm{d} S_{S} / \mathrm{d} T<0$ and solidification within the frozen fringe acts to reduce the rate at which the temperature changes in comparison with what would be expected if latent heat effects were ignored. This causes much of the variations in temperature gradient responsible for the deviations in $\psi$ from unity. The advective term in equation (12) neglects the small variations to the rate of upward ice motion through the fringe that are related to the density difference between liquid water and ice.

[28] To solve the second-order partial differential equations for temperature in each of the three regions, as well as the positions of the two moving boundaries, I need to specify a total of eight boundary conditions. In addition to the fixed temperature conditions at the surface and $z_{f}$, the far-field temperature as $z \rightarrow \infty$ is also constant, at $T_{i}$. Matching temperature conditions apply across the two moving boundaries. The heat flux conditions at $z_{f}$ and $z_{l}$ are

$$
\begin{gathered}
\left.\frac{\partial T}{\partial z}\right|_{z_{l}^{+}}-\left.\frac{\partial T}{\partial z}\right|_{z_{l}^{-}}=-\frac{\mathcal{L}}{\kappa \mathcal{C}}\left[1-\phi S_{S}\left(T_{l}\right)\right]\left(\mathbf{V}_{l} \cdot \hat{\mathbf{z}}\right) \\
\left.\frac{\partial T}{\partial z}\right|_{z_{f}^{+}}-\left.\frac{\partial T}{\partial z}\right|_{z_{f}^{-}}=-\frac{\mathcal{L}}{\kappa \mathcal{C}} \phi S_{s}\left(T_{f}\right) \frac{\mathrm{d} z_{f}}{\mathrm{~d} t},
\end{gathered}
$$

where $\mathbf{V}_{l} \cdot \hat{\mathbf{z}} \equiv \mathrm{d} z_{l} / \mathrm{d} t$, and the superscripts indicate on which side of the boundaries the temperature gradients are evaluated. For the saturation profile dictated by equation (10) $S_{s}\left(T_{f}\right)=0$ and the right side of equation (14) vanishes. The final condition is supplied by the mechanical constraint embodied in equation (6). As part of the solution procedure, equation (8) is used to check whether the particle pressure $P_{p}$ reaches zero so that a new lens can form. I do not examine the dynamics of lens initiation in detail, but assume that the new lens forms quickly at the location where $P_{p}$ first equals zero and acts as an effective barrier to further fluid supply to the overlying region. Hence the subsequent heave is determined by fluid supply only to the newly developed lens.

\subsection{Transformation to Stretched Coordinates}

[29] For the classic Stefan problem describing the motion of a solidification front through a uniform half-space, a similarity solution results in which the position of the moving phase boundary is expected to increase with the square root of time [e.g., Wettlaufer, 2001]. For the current problem, the temperature at $z_{l}$ is not constant, so I do not expect a straightforward similarity solution with $z_{l} \propto \sqrt{t}$. However, the treatment is simplified by focusing on deviations from the behavior of the classical Stefan problem and hence it is convenient to make a variable transformation to something resembling similarity coordinates. Accordingly, I define $\xi \equiv z /(2 \sqrt{\kappa t})$ and $\tau \equiv t$, and let $z_{l}=2 \lambda_{l}(\tau) \sqrt{\kappa t}$ and $z_{f}=2 \lambda_{f}(\tau) \sqrt{\kappa t}$, so that the transformed problem becomes

$$
4 \tau \frac{\partial T}{\partial \tau}=\frac{\partial^{2} T}{\partial \xi^{2}}+2 \xi \frac{\partial T}{\partial \xi} ; \lambda_{l}(\tau)>\xi>0
$$




$$
\begin{aligned}
& 4 \tau \frac{\partial T}{\partial \tau}\left(1-\frac{\mathcal{L} \phi}{\mathcal{C}} \frac{\mathrm{d} S_{s}}{\mathrm{~d} T}\right)=\frac{\partial^{2} T}{\partial \xi^{2}} \\
& \quad+\left(2 \xi-2 \lambda_{l}-4 \tau \frac{\mathrm{d} \lambda_{l}}{\mathrm{~d} \tau}\right) \frac{\partial T}{\partial \xi}\left(1-\frac{\mathcal{L} \phi}{\mathcal{C}} \frac{\mathrm{d} S_{s}}{\mathrm{~d} T}\right) ; \lambda_{f}(\tau)>\xi>\lambda_{l}(\tau)
\end{aligned}
$$

$$
4 \tau \frac{\partial T}{\partial \tau}=\frac{\partial^{2} T}{\partial \xi^{2}}+2 \xi \frac{\partial T}{\partial \xi} ; \xi>\lambda_{f}(\tau)
$$

subject to the heat flux conditions implied by equations (13) and (14), namely,

$$
\begin{array}{r}
\left.\frac{\partial T}{\partial \xi}\right|_{\lambda_{l}^{+}}-\left.\frac{\partial T}{\partial \xi}\right|_{\lambda_{l}^{-}}=-\frac{\mathcal{L}}{\mathcal{C}}\left[1-\phi S_{S}\left(T_{l}\right)\right]\left(4 \tau \frac{\mathrm{d} \lambda_{l}}{\mathrm{~d} \tau}+2 \lambda_{l}\right) \\
\left.\frac{\partial T}{\partial \xi}\right|_{\lambda_{f}^{+}}-\left.\frac{\partial T}{\partial \xi}\right|_{\lambda_{f}^{-}}=-\frac{\mathcal{L}}{\mathcal{C}} \phi S_{S}\left(T_{f}\right)\left(4 \tau \frac{\lambda_{f}}{\mathrm{~d} \tau}+2 \lambda_{f}\right),
\end{array}
$$

the initial condition $T(\xi, 0)=T_{i}$, boundary conditions $T(0$, $\tau)=T_{s}, T\left(\lambda_{f}, \tau\right)=T_{f}, T(\infty, \tau)=T_{i}$, and continuity in temperature at $\xi=\lambda_{l}(\tau)$ and $\lambda_{f}(\tau)$. To close the problem, I transform equation (6) and write the mechanical constraint on the lens position as

$$
4 \tau \frac{\mathrm{d} \lambda_{l}}{\mathrm{~d} \tau}=\frac{H\left(T_{l}\right) \psi\left(\lambda_{l}, T_{l}\right)}{\kappa\left(\lambda_{f}-\lambda_{l}\right)}-2 \lambda_{l},
$$

where

$$
\psi(\xi, T)=\left(\frac{\lambda_{f}-\lambda_{l}}{T_{f}-T_{l}}\right) \frac{\int_{T}^{T_{f}}\left[\left(1-\phi S_{s}\right)^{2} / k\right] \mathrm{d} T}{\int_{\xi}^{\lambda_{f}}\left[\left(1-\phi S_{s}\right)^{2} / k\right] \mathrm{d} \xi} .
$$

The manner in which this system of equations is used to determine the temperature profile in the stretched coordinates is illustrated in Figure 5.

\subsection{Freezing Regimes}

[30] Equations (15) through (17), together with the initial condition and boundary conditions summarized above, dictate the system evolution between episodes of lens initiation. To predict the boundary temperature on the first of these lenses in the step freezing configuration considered here, I begin by solving a special case of the mechanical constraint on lens initiation embodied in equation (7). As with subsequent initiation events, the first lens forms where $P_{p}=0$. However, as noted earlier, with no preexisting lens to induce liquid migration, the fluid pressure distribution is initially hydrostatic; $\mathbf{V}_{l}=0$ and the second term in equation (7) contributes nothing to the force balance. Accordingly, the lens temperature $T_{l}\left(0^{+}\right)$an instant after the surface temperature is imposed is found as the solution to

$$
P_{0}=\frac{\rho \mathcal{L}}{T_{m}}\left[\phi S_{s}\left(T_{m}-T\right)-\int_{T}^{T_{f}} \phi S_{s} \mathrm{~d} T\right] .
$$

This corresponds to the condition that $H_{c}\left(T, T_{l}\right)=0$.

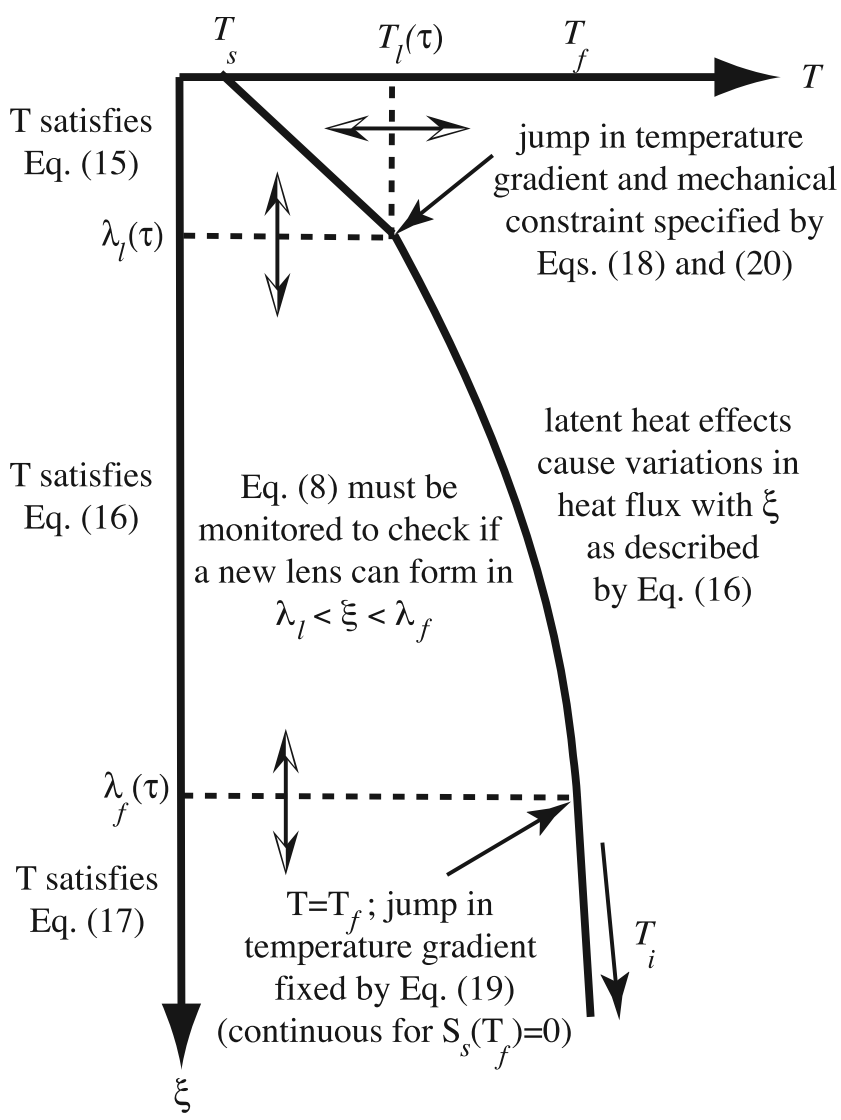

Figure 5. Schematic diagram showing the temperature profile expected in the stretched coordinates for a stepfreezing configuration and the considerations involved in obtaining its solution.

[31] Since the surface temperature $T_{s} \leq T_{l}$ equation (21) implies that lenses can only form when

$$
P_{0} \leq P_{\max }\left(T_{s}\right) \equiv \frac{\rho \mathcal{L}}{T_{m}}\left[\phi S_{s}\left(T_{m}-T_{s}\right)-\int_{T_{s}}^{T_{f}} \phi S_{s} \mathrm{~d} T\right],
$$

where it is understood that the first $S_{s}$ is evaluated at $T_{s}$. $P_{\max }$ is identified as the maximum frost heave pressure for a porous medium with ice saturation profile $S_{s}$ and porosity $\phi$; this marks one boundary between different freezing regimes. When $P_{0}>P_{\max }$ ice is expected to form only within the pore space without causing deformation to the ground surface. The evolution of temperature and the fringe boundary position in this limit satisfy a subset of the equations given above, but without a defined $\lambda_{l}$ so that no $\tau$ variations occur and $\lambda_{f}$ is constant as a result.

[32] Figure 6 shows the maximum frost heave pressure as a function of surface temperature for the three different porous media types characterized in Table 1. A formula for $P_{\max }$ that makes use of equations (9) and (10) for $k$ and $S_{s}$ is given in Appendix A. The case of the idealized porous medium that is characterized by melt channels of the size expected to result from the Gibbs-Thomson effect exhibits a maximum frost heave pressure that approaches a limiting value at low surface temperatures that is indicated with the dotted line. The Chena silt and Inuvik clay both have 


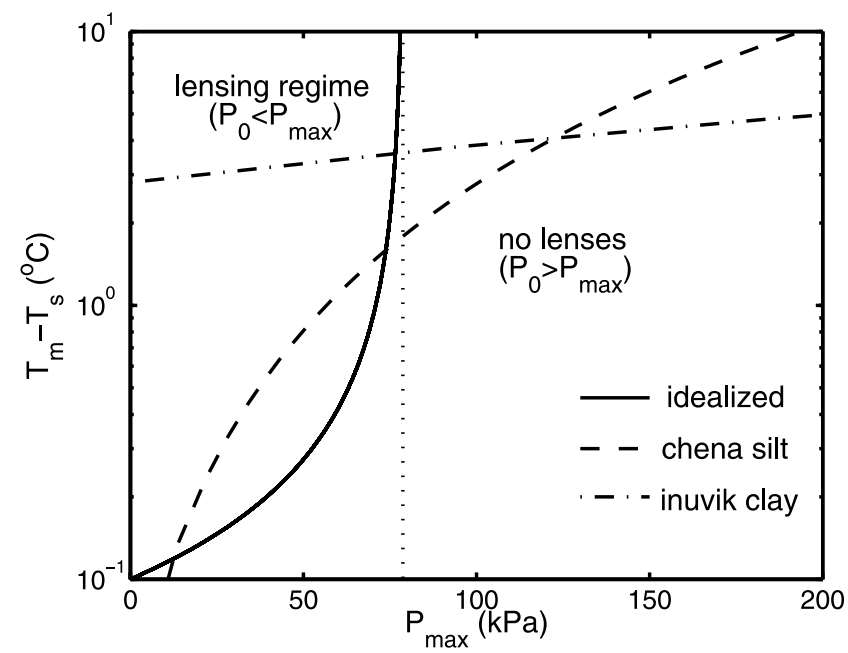

Figure 6. Variation in maximum frost heave pressure with surface temperature for the parameters summarized in Table 1. Lenses are expected to be initiated only for $P_{0}<P_{\max }$.

predicted maximum frost heave pressures that increase indefinitely as the surface temperature is decreased. The use of a more complicated empirical formula for $S_{s}$ with an additional temperature-dependent term to account for changes in the relative importance of the Gibbs-Thomson effect in comparison with the interactions that lead to interfacial premelting would be expected to yield qualitative behavior that is intermediate between that exhibited by the solid and dashed curves; the former being more reliable at warmer temperatures and the latter at colder temperatures.

[33] With the initial value of $T_{l}$ determined by (21), I next solve equations (15) through (17) for the temperature profile and the positions of the fringe and lens boundaries an instant after the surface temperature is imposed. In the limit that $\tau \rightarrow 0^{+}$, the physical distance over which the temperature increases from $T_{s}$ at the surface to the initial uniform soil temperature $T_{i}$ is infinitesimally small, but the stretched coordinate $\xi$ representing this vanishingly small separation spans the entire range of positive numbers from 0 to $\infty$. Assuming that the continuum description of heat flow remains a good approximation for the system behavior at this early time, I seek the values of $\xi=\lambda_{l}$ and $\xi=\lambda_{f}$ at which the temperatures are $T_{l}$ and $T_{f}$ respectively when $t=$ $0^{+}$. The temperature profiles above the lens and in the ice free regions are

$$
\begin{array}{r}
T\left(\lambda_{l}>\xi>0,0^{+}\right)=\frac{T_{l}-T_{s} \operatorname{erfc} \lambda_{l}}{\operatorname{erf} \lambda_{l}} \operatorname{erf} \xi+T_{s} \operatorname{erfc} \xi \\
T\left(\xi>\lambda_{f}, 0^{+}\right)=T_{i} \operatorname{erf} \xi+\frac{T_{f}-T_{i} \operatorname{erf} \lambda_{f}}{\operatorname{erfc} \lambda_{f}} \operatorname{erfc} \xi,
\end{array}
$$

where $\operatorname{erf} u \equiv(2 / \sqrt{\pi}) \int_{0}^{u} \exp \left(-v^{2}\right) \mathrm{d} v$ and $\operatorname{erfc} u \equiv 1-\operatorname{erf} u$ are the error and complementary error functions [e.g., Abramowitz and Stegun, 1964]. The temperature profile through the fringe and the initial values of $\lambda_{l}$ and $\lambda_{f}$ are found by solving

$$
0=\frac{\mathrm{d}^{2} T}{\mathrm{~d} \xi^{2}}+2\left(\xi-\lambda_{l}\right) \frac{\mathrm{d} T}{\mathrm{~d} \xi}\left(1-\frac{\mathcal{L} \phi}{\mathcal{C}} \frac{\mathrm{d} S_{s}}{\mathrm{~d} T}\right)
$$

subject to the heat flux conditions

$$
\begin{gathered}
\left.\frac{\mathrm{d} T}{\mathrm{~d} \xi}\right|_{\lambda_{l}^{+}}=\frac{2}{\sqrt{\pi}} e^{-\lambda_{l}^{2}} \frac{T_{l}-T_{s}}{\operatorname{erf} \lambda_{l}}-\frac{\mathcal{L}}{\mathcal{C}}\left[1-\phi S_{s}\left(T_{l}\right)\right] \frac{H\left(T_{l}\right) \psi\left(\lambda_{l}, T_{l}\right)}{\kappa\left(\lambda_{f}-\lambda_{l}\right)} \\
\left.\frac{\mathrm{d} T}{\mathrm{~d} \xi}\right|_{\lambda_{f}^{-}}=\frac{2}{\sqrt{\pi}} e^{-\lambda_{f}^{2}} \frac{T_{i}-T_{f}}{\operatorname{erfc} \lambda_{f}}+\frac{\mathcal{L}}{\mathcal{C}} \phi S_{s}\left(T_{f}\right) 2 \lambda_{f} .
\end{gathered}
$$

and the known boundary temperatures $T\left(\lambda_{f}\left(0^{+}\right)\right)=T_{f}$ and $T\left(\lambda_{l}\left(0^{+}\right)\right)=T_{l}\left(0^{+}\right)$. Equations (18) and (20) have been combined in equation (26); $S_{s}\left(T_{f}\right)=0$ for saturation profiles of the form given by equation (10) so the second term on the right side of (27) is zero. I use a shooting method to solve for $\lambda_{f}\left(0^{+}\right), \lambda_{l}\left(0^{+}\right)$and $T\left(\lambda_{f}>\xi>\lambda_{l}, 0^{+}\right)$, with $\lambda_{l}$ as the shooting parameter and the convergence criterion requiring that $T\left(\lambda_{f}\right)=T_{f}$ be satisfied to within a specified tolerance when the temperature gradient satisfies equation (27).

[34] Having found the starting positions of the lens and fringe boundaries, I next examine how these evolve through time. Equation (20) suggests three possibilities for the motion of the lens, as illustrated with a double-sided arrow in Figure 5. (1) If $H\left(T_{l}\right) \psi\left(\lambda_{l}, T_{l}\right)>2 \kappa \lambda_{l}\left(\lambda_{f}-\lambda_{l}\right)$, then $\mathrm{d} \lambda_{l} / \mathrm{d} \tau>0$ and the lens advances more rapidly than the square root of time. I interpret this to mean that the release of latent heat occurs at a faster rate than it can be conducted away from the lens boundary so I expect the lens temperature to increase initially; eventually this can cause the lens temperature to increase beyond $T_{f}$ so that the fringe disappears and only the single lens grows, with no further initiation. (2) If $H\left(T_{l}\right) \psi\left(\lambda_{l}, T_{l}\right)<2 \kappa \lambda_{l}\left(\lambda_{f}-\lambda_{l}\right)$, then $\mathrm{d} \lambda_{l} / \mathrm{d} \tau<0$ and I expect the lens temperature to decrease initially; as the fringe thickens and the lens migrates toward colder temperatures, the pore space can become sufficiently filled with ice that a new lens initiates at a warmer temperature so that periodic lensing results. 3) If $H\left(T_{l}\right) \psi\left(\lambda_{l}, T_{l}\right)=2 \kappa \lambda_{l}\left(\lambda_{f}-\lambda_{l}\right)$, then $\mathrm{d} \lambda_{l} / \mathrm{d} t=0$ and the system satisfies a similarity solution, which greatly simplifies the mathematics by removing all the time derivatives in the governing equations. However, this solution is not stable to perturbations in $\lambda_{l}$, so it is expected to eventually relax into the behavior described above for cases 1 or 2. Instead, this special case is of particular interest because it is expected to mark the boundary between the single and multiple lens regimes.

[35] Figure 7 shows the boundary between different freezing regimes for the idealized porous medium. The solid curve marks the minimum surface temperature that permits lenses to be initiated so that heaving can occur. Within the lensing regime, when the fluid is initially stagnant the solid line also gives the temperature of the first lens at its initiation. Using equation (6) to calculate the capacity for heaving of this first lens $H\left(T_{l 1}\right)$, the dash-dotted curve indicates conditions under which the mechanical constraint on the lens position given by equation (20) is 


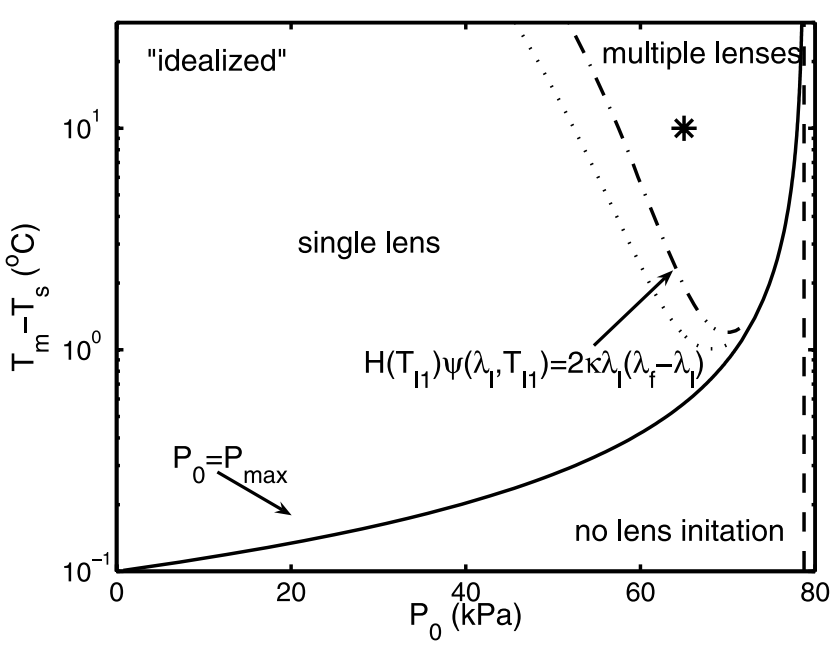

Figure 7. Regime diagram showing conditions under which different types of freezing behavior are predicted within a porous medium characterized by the parameters listed in the first column of Table 1. The solid line shows the maximum frost heave pressure defined by equation (22), plotted against the temperature depression from bulk melting at the surface $T_{m}-T_{s}$. Lens initiation can occur only when $P_{0} \leq P_{\max }$. The temperature at the boundary of the first lens falls on this solid curve, and its capacity for heaving is given by equation (6). The dash-dotted curve marks conditions for which $H\left(T_{l 1}\right) \psi\left(\lambda_{l}, T_{l 1}\right)=2 \kappa \lambda_{l}\left(\lambda_{f}-\lambda_{l}\right)$ and the rate of heat and mass diffusion in the assumed stepfreezing configuration would permit a constant lens temperature. This is not a stable state, however. Colder surface temperatures cause the lens temperature to decrease, and multiple lenses eventually form, whereas warmer surface temperatures produce an initial period of adjustment followed by the stable growth of a single lens with a constant boundary temperature. The vertical dashed line shows the asymptotic value of $P_{\max }$ in the low surface temperature limit. The point labeled with the asterisk represents the conditions used to generate Figure 9.

met with a steady value of $\lambda_{l}$ in the stretched coordinate frame. Below this curve $H\left(T_{l}\right) \psi\left(\lambda_{l}, T_{l 1}\right)>2 \kappa \lambda_{l}\left(\lambda_{f}-\lambda_{l}\right)$ and the lens temperature is expected to increase initially before it reaches a steady configuration that is stable. This is the regime in which needle ice grows near the ground surface, typically with ice-free sediments below and the tops of the ice needles supporting only very small surface loads, such as pebbles or pieces of bark mulch. Above the dash-dotted curve the lens temperature decreases and the fringe ice content grows until a new lens is initiated eventually at a slightly cooler temperature than the first lens, as illustrated for $P_{0}=65 \mathrm{kPa}$ in Figures 3 and 4 . This is the regime in which periodic lensing occurs. For comparison, the dotted curve corresponds to $H\left(T_{l}\right)=2 \kappa \lambda_{l}\left(\lambda_{f}-\lambda_{l}\right)$, which would be expected to mark the boundary between the single and periodic lensing regimes if the fringe temperature gradient were uniform (e.g., $\psi\left(\lambda_{l}, T_{l}\right)=1$ ). The maximum frost heave pressure depends only on the temperature at the lens boundary and not the details of the temperature profile beneath.
[36] In Figure 8, regime diagrams are given for Chena silt and Inuvik clay. In comparison to the idealized case shown in Figure 7, in Chena silt the multiple lensing regime extends to much lower overburden pressures. The single lens regime is inhibited by lower permeabilities, which reduce the rate of fluid supply so that the release of latent heat that accompanies lens growth cannot keep pace with the rate of heat transport. For Inuvik clay, the single lens regime is practically absent, with the dash-dotted curve marking conditions where $H\left(T_{l}\right) \psi\left(\lambda_{l}, T_{l 1}\right)=2 \kappa \lambda_{l}\left(\lambda_{f}-\lambda_{l}\right)$ lying just slightly above the solid curve for which $P_{0}=$ $P_{\max }$, so that the former curve is almost completely obscured at this scale.

[37] Though our analysis was based on the assumption of constant overburden $P_{0}$ and surface temperature $T_{s}$, it is instructive to refer to the regime diagrams in Figures 7 and 8 for intuition into the expected system behavior under transient conditions. For instance, consider a constant surface temperature of $T_{m}-T_{s}=1{ }^{\circ} \mathrm{C}$, but an increasing surface load $P_{0}$, as would be expected for freezing at the ground surface with no added weight. For both the idealized porous medium and the Chena silt, the regime diagrams indicate that freezing would begin in the single lens regime. As the overburden increases, the system moves into the multiple lens regime and then eventually the regime in which no lenses are initiated. This is the qualitative behavior one expects. It should be emphasized, however, that transitions between the single and the multiple lens regimes are expected to be offset from the regime boundaries shown here, which derive from a comparison between the rate of latent heat release from the first lens at its inception with the rate of heat flow. To go from the single to the multiple lens regime requires that heat be removed more rapidly than latent heat is released at the maximum fluid supply rate possible to the lens boundary; for the idealized porous medium this coincides with the lens temperature at the peak in $H\left(T_{l}\right)$ shown in Figure 4 (e.g., $T_{m}-T_{l} \approx 0.12^{\circ} \mathrm{C}$ ). To go from the multiple lens regime to the single lens regime requires that the rate of latent heat release at the inception of a new lens exceed the rate of heat removal.

[38] In this section, I have focused on determining the overall character, or phase of behavior to be expected from a soil with known characteristics (i.e., $S_{s}$ and $k$ given as a function of $T$ ) that is subjected to a particular set of environmental conditions (i.e., fixed surface temperature $T_{s}$, overburden $P_{0}$ ). Regime diagrams of the type given in Figures 7 and 8 can be readily constructed for other porous media in a step-freezing configuration by following the procedure presented here. Moreover, the maximum frost heave pressure, given by equation (22), is insensitive to the structure of the thermal field beneath the incipient lens and so can be applied to delineate the requirements for lens formation under other thermal conditions, in addition to the step-freezing configuration considered here. The location of the boundary between the multiple lensing and single lensing regimes is moderately sensitive to the temperature field within the fringe, as illustrated by the differences between the dotted and dash-dotted curves in Figure 7. However, the heaving capacity $H$ acts as the primary control on the type of lensing behavior that is expected to arise and the variations in temperature gradient that cause $\psi$ to deviate from unity are of much lesser importance. For the step- 

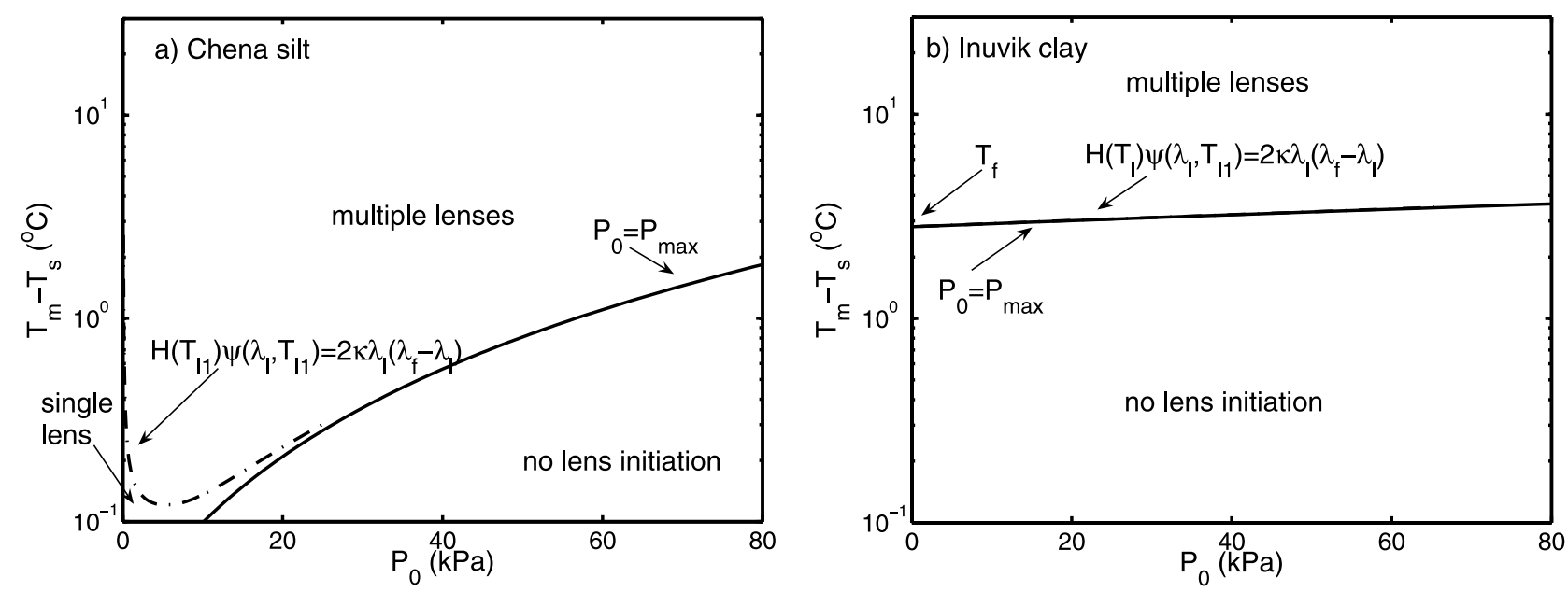

Figure 8. Regime diagrams showing conditions under which different types of freezing behavior are predicted within (a) Chena silt and (b) Inuvik clay, as characterized by the parameters listed in Table 1.

freezing configuration the regime boundary between the single and multiple-lensing regimes represents a steady state, albeit an unstable one, in which the governing equations admit a similarity solution with no contribution from the terms involving time derivatives in equations (15) through (20). Having determined where these regime boundaries lie, in the following section I examine briefly the more challenging problem of predicting the system evolution and macroscopic behavior within the multiplelensing regime.

\subsection{Approximate Solutions for Periodic Lensing}

[39] Figure 9 shows the calculated early temperature profile in the stretched coordinate frame for the parameter choices summarized in the first column of Table 1, with $P_{0}=$ $65 \mathrm{kPa}$. The initial values of $\lambda_{f}$ and $\lambda_{l}$ are marked with the labeled squares. Temperature conditions were set so that $T_{i}=$ $T_{m}, T_{m}-T_{s}=10^{\circ} \mathrm{C}$, and $H\left(T_{l}\right) \psi\left(\lambda_{l}, T_{l}\right)<2 \kappa \lambda_{l}\left(\lambda_{f}-\lambda_{l}\right)$. Hence I expect periodic lensing to result, as indicated on the regime diagram of Figure 7 by the asterisk, which corresponds to the modeled conditions. Either a slight decrease in the overburden from $P_{0}=65 \mathrm{kPa}$ to $55 \mathrm{kPa}$, or an increase in the surface temperature so that $T_{m}-T_{S}<1.9^{\circ} \mathrm{C}$, results in $H\left(T_{l}\right) \psi\left(\lambda_{l}, T_{l}\right)>2 \kappa \lambda_{l}\left(\lambda_{f}-\lambda_{l}\right)$. In such circumstances, the lens temperature is expected to increase with time, leading eventually to the prolonged growth of a single lens, in this case without an underlying frozen fringe.

[40] Lens spacing and thickness can be predicted by solving the mathematical problem described by equations (15)-(20). However, the time dependence complicates matters, and here I present only an approximate treatment instead. Figure 10 shows the lens and fringe coordinates obtained for the idealized porous medium using a quasistatic approximation in which I use the initial profile from equation (23) to describe temperatures above the lens boundary. Since the mechanical constraint given by equation (20) indicates that $\tau \mathrm{d} \lambda_{l} / \mathrm{d} \tau$ is negative, I adjust the lens position by a small increment toward smaller values and assign the corresponding lens temperature using equation (23). I use this temperature and the gradient given by equation (26) as initial conditions to integrate equation (25) until reaching $T_{f}$, at which point I assign the new fringe coordinate $\lambda_{f}$. The value of $\psi\left(\lambda_{l}, T_{l}\right)$ is then updated before another increment in $\lambda_{l}$ is taken and the procedure is repeated. Once the mechanical condition for lens initiation from equation (8) is satisfied somewhere in the interior of the fringe, I note the location of the previous lens at its maximum extent and the location at which the new lens is expected to be formed.

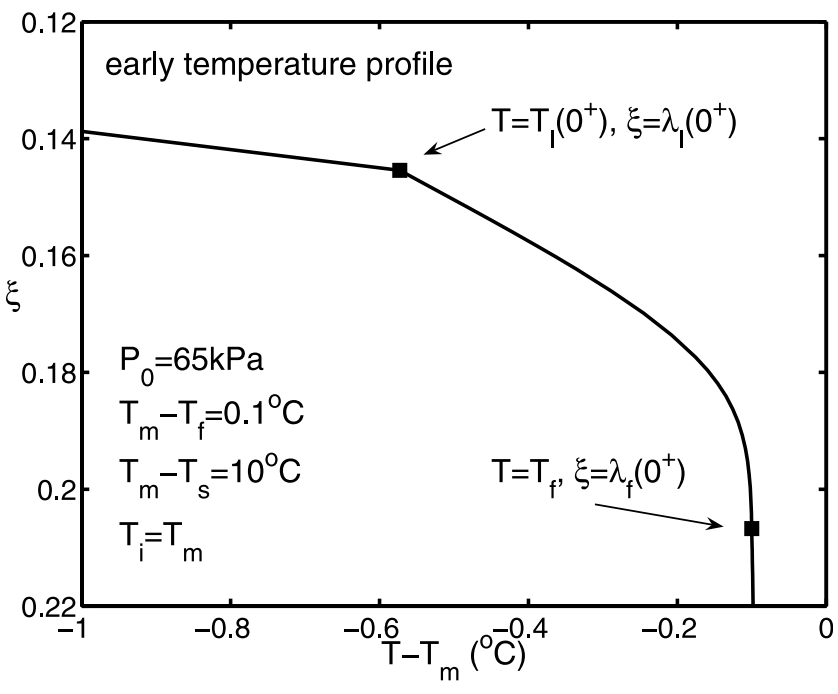

Figure 9. Early temperature profile, immediately following the initiation of the first lens. For the parameters chosen here, the lens growth condition is $2 \kappa \lambda_{l}\left(\lambda_{f}-\lambda_{l}\right) \approx$ $0.012 \mathrm{~mm}^{2} / \mathrm{s}$, shown with a dashed line in Figure 4 . The deviation of the fringe temperature profile from a linear gradient leads to $\psi\left(\lambda_{l}, T_{l}\right) \approx 1.79$ for this case, so that $2 \kappa \lambda_{l}\left(\lambda_{f}-\lambda_{l}\right) / \psi\left(\lambda_{l}, T_{l}\right) \approx 0.0070 \mathrm{~mm}^{2} / \mathrm{s}$. As shown by the dotted line in Figure 4 , this exceeds $H\left(T_{l}\right) \approx 0.0022 \mathrm{~mm}^{2} / \mathrm{s}$ (the asterisk labeled " 1 st"), and I expect the lens temperature to subsequently decrease so that periodic lensing results. The conditions used for this calculation correspond to the point labeled with an asterisk in the regime diagram of Figure 7. 


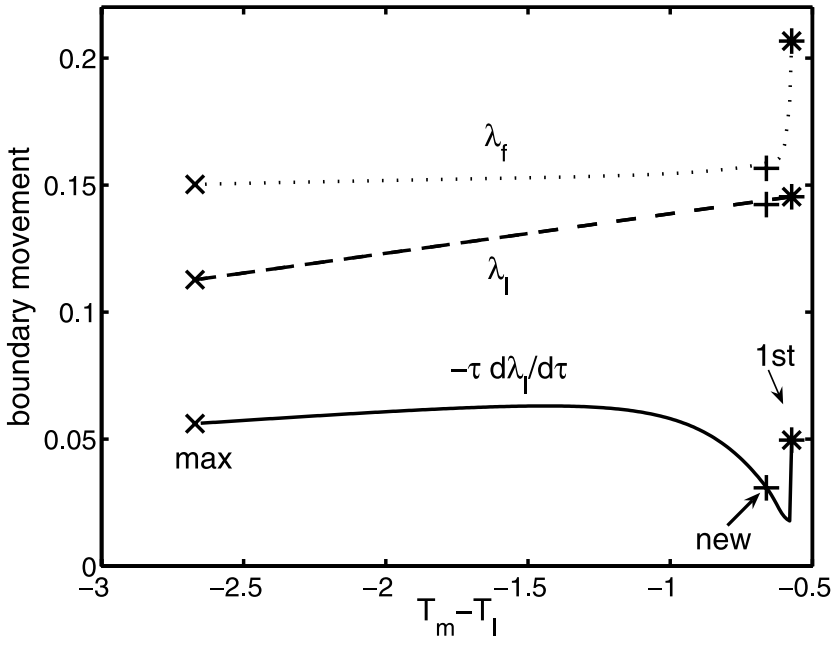

Figure 10. Lens $\lambda_{l}$ and fringe $\lambda_{f}$ coordinates in the stretched reference frame and the scaled rate of lens boundary motion $-\tau \mathrm{d} \lambda_{l} / \mathrm{d} \tau$. The conditions used for this calculation correspond to the point labeled with an asterisk in the regime diagram of Figure 7.

These are labeled in Figure 10, where I also show the scaled rate of motion for the lens coordinate $-\tau \mathrm{d} \lambda_{l} / \mathrm{d} \tau$.

[41] The approximate spacing between lenses is given by the difference between the position at which a new lens would be initiated at time $\tau$ and the position of the previous lens at its maximum extent. This is simply calculated as

$$
\operatorname{spacing}(\tau) \approx 2\left(\lambda_{l n}-\lambda_{l m}\right) \sqrt{\kappa \tau}
$$

where the lens positions in stretched coordinates are $\lambda_{l n}$ and $\lambda_{l m}$ at initiation and maximum extent. The approximate lens thickness is calculated as the difference between the lens position at its maximum extent, and the lens position a time $\Delta \tau$ earlier, when it was first initiated. Using equation (20), I approximate this time difference as

$$
\Delta \tau \approx \tau\left[1-\exp \left(\int_{\lambda_{l n}}^{\lambda_{l m}} \frac{-4 \kappa\left(\lambda_{f}-\lambda_{l}\right)}{H\left(T_{l}\right) \psi\left(\lambda_{l}, T_{l}\right)-2 \lambda_{l} \kappa\left(\lambda_{f}-\lambda_{l}\right)} \mathrm{d} \lambda_{l}\right)\right]
$$

where the integrand is simply $f\left(\lambda_{l}\right)=\left(-\tau \mathrm{d} \lambda_{l} / \mathrm{d} \tau\right)^{-1}$. Figure 10 indicates that $f\left(\lambda_{l}\right)$ does not change appreciably over the course of lens growth, so I could also write $\Delta \tau \approx \tau-$ $\tau \exp \left[\left(\lambda_{l m}-\lambda_{l n}\right) \bar{f}\right]$, where $\bar{f}$ is the average value of $f\left(\lambda_{l}\right)$. The thickness of a lens that has just stopped growing at time $\tau$ is

$$
\text { thickness }(\tau) \approx 2 \lambda_{m} \sqrt{\kappa \tau}-2 \lambda_{n} \sqrt{\kappa(\tau-\Delta \tau)} .
$$

The approximate depth to the base of the lowermost active lens is

$$
\operatorname{depth}(\tau) \approx\left(\lambda_{l n}+\lambda_{l m}\right) \sqrt{\kappa \tau} .
$$

I approximate the total extent of heave by weighting this depth with the ratio of the lens thickness to the sum of the lens thickness and spacing so that

$$
\operatorname{heave}(\tau) \approx \operatorname{depth}(\tau) \frac{\operatorname{thickness}(\tau)}{\operatorname{thickness}(\tau)+\operatorname{spacing}(\tau)} .
$$

Heave dimensions calculated in this way are shown in Figure 11 for the idealized medium.

[42] Table 2 summarizes the predicted boundary positions for all three porous media using the same surface temperature, initial temperature, and overburden. For the Chena silt, the lens and fringe positions reported in Table 2 are considerably different from those for the idealized medium. The arguments given above lead to predicted lens spacings that are greater than those for the idealized medium, but the thicknesses of these lenses are smaller so the accumulated heave is less than a millimeter, even after 100 hours under these conditions. For the Inuvik clay, as shown in Figure $8 \mathrm{~b}$, with $P_{0}=65 \mathrm{kPa}$ a surface temperature of $T_{s}=-10^{\circ} \mathrm{C}$ is sufficiently cold that the rate of heat flow exceeds the rate of fluid supply once the first lens forms; this is the condition that I use to delineate the boundary between the single and multiple lensing regimes. The lens temperature is expected to decrease thereafter, but for this case the surface temperature is still too warm to allow the fringe to cool sufficiently that a new lens can be initiated. Neglecting the effects of nonlinearities in the temperature profile, I can find the temperature $T_{\text {new }}$ at which a new lens is initiated once the old lens reaches temperature $T_{\max }$ by seeking the solution to $H_{c}\left(T_{\text {new }}, T_{\max }\right)=H\left(T_{\max }\right)$, where $H_{c}\left(T=T_{\text {new }}, T_{\max }\right)$ is at a maximum with respect to variations in $T$. I find that new lenses are expected to form with $T_{m}-T_{\text {new }} \approx 5.06^{\circ} \mathrm{C}$ only once the temperature at the old lens reaches $T_{m}-T_{\max } \approx$ $64.9^{\circ} \mathrm{C}$. The lens positions and value of $\psi$ reported in Table 2 for $T_{m}-T_{l}=5.06^{\circ} \mathrm{C}$ are those expected when

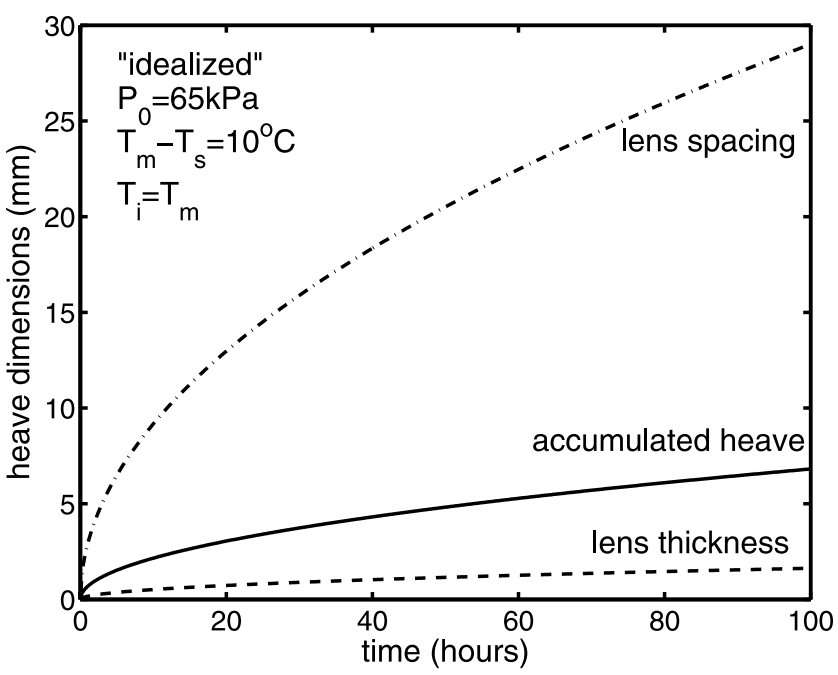

Figure 11. Approximate lens spacing, thickness, and accumulated heave as a function of time. Parameter values are summarized in the first column of Table 1, while the boundary locations and temperatures are given in Table 2. 
the surface temperature is $T_{m}-T_{s}=10^{\circ} \mathrm{C}$ and the first lens reaches an undercooling of $5.06^{\circ} \mathrm{C}$. Over time this lens continues to cool until it approaches the surface temperature of $T_{m}-T_{s}=10^{\circ} \mathrm{C}$, as $\lambda_{l}$ decreases toward zero and $\lambda_{f}$ tends to a long-term limit of $\lambda_{f} \approx 0.476$.

\section{Discussion}

[43] I have presented what might be considered as a minimal model for the dynamics of ice formation within porous media subjected to transient freezing. I have included only those effects that are essential to determining how the solidification morphology develops, whether the system attains the single lens, multiple lensing, or pore freezing regimes. Though I expect the predictions to be robust in a qualitative sense, there are many additional complicating factors that can significantly modify the behavior of natural systems. Some of these, such as differences between the thermal properties of the system components, would require little further effort to incorporate within a more complete description, but are neglected here primarily to avoid distracting attention from the dominant physical effects. Similarly, with the availability of sufficient empirical constraints, it would be straightforward to use more sophisticated ice saturation and permeability parameterizations that are designed to reflect the effect of temperature decreases on the relative importance to the fluid network of surface energy and interfacial premelting. Several other physical effects that have been neglected here could be included within the framework of a revised model that would be considerably more complex. I briefly outline a few of these here, then comment on the effects of a couple of the approximations made in the present model, before concluding.

[44] The effect of freeze-thaw cycles in altering the susceptibility to lens formation and heave of pristine porous media is a common practical consideration in engineering studies [e.g., Andersland and Ladanyi, 2004]. More generally, such changes to the ice saturation behavior of soils is expected to occur in large part as a result of poroelastic effects and inelastic dilatancy. Indeed, anecdotal reports of sediment compaction near the lens boundary (J. S. Wettlaufer, personal communication, 2000) are consistent with large increases in interparticle pressures close to the growing lens predicted by equation (7). As noted in section 2.4 , one approach to generalizing the analysis to include poroelastic effects would be to treat the porosity $\phi$ as a function of effective stress $P_{p}$, as is commonly observed in studies of fluid-infiltrated porous media that are above the melting temperature. I expect further experimental efforts to soon yield much better constraints on the manner in which to proceed on this front. Inelastic effects that cause permanent changes to soil microstructures are somewhat more challenging to quantify. In some cases these may be related to the chemical changes in clays that are known to occur upon freezing on the basis of the observed degradation to the surface properties that are necessary for the fabrication of ceramics (G. Ngan, personal communication, 2005). The movement and concentration of solutes in the unfrozen pore water is likely to play a large role in these changes.

[45] Recent attention has been drawn to the potential for segregated ice growth as a contributing mechanism to the incorporation of sediments in the basal regions of glaciers [Christoffersen and Tulaczyk, 2003]. This intriguing possibility has been bolstered by borehole photographs of sediment bands beneath an Antarctic ice stream [Carsey et al., 2002]. Interest is driven primarily by the recognition that glacier motion in these fast-flowing regions is largely accomplished by till deformation beneath the ice front and the expectation that the shear resistance in these sediments is dependent on the effective stress. In the formulation presented here I have treated the case where the effective stress at the furthest extent of pore ice $P_{0}$ is constant. This may not be at all appropriate beneath glaciers, where the hydrological systems that operate are notoriously complex. Moreover, the thermal regime beneath a thick insulating layer of glacial ice will typically be characterized by much more shallow temperature gradients than those considered here, and the importance of deformational heating does complicate the heat balance. Nevertheless, even with these considerations, the formulation of a rigorous predictive model for the characteristics of basal freeze-on beneath glacial ice should not represent an overly ambitious extension to the present work.

[46] Textbook descriptions of the weathering of cohesive materials by frost action commonly focus on the modest volumetric expansion that accompanies the phase transition. Persuasive arguments by Walder and Hallet [1986], together with laboratory [Hallet et al., 1991; Murton et al., 2006] and field studies [Matsuoka, 2001; Hales and Roering, 2005] suggest that most frost damage actually occurs as a result of the same premelting dynamics as control the segregated ice growth described here. Walder and Hallet [1985] present a model that examines the extension of preexisting cracks in cohesive materials, based on the frost heave formulation of Gilpin [1980]. A more complete description of landscape evolution driven by frost weathering must account for environmental controls on the fluid supply through fractures and joints to the freezing centers, as well as the likely importance in many field situations of partial air saturation. Moreover, the anisotropic stress distributions that characterize steep slope exposures and the potential insulating effects of snow accumulations on shallow exposures also need to be considered.

[47] I have chosen a system in which the properties and environmental forcing are assumed to be laterally continuous. Differential frost heave in nature occurs where lateral variations are significant, whether they are initially imposed or develop spontaneously in response to feedback mechanisms. Much has been learned about these systems from studies [Kessler and Werner, 2003; Peterson and Krantz, 2003; Plug and Werner, 2001, 2002; Sletten et al., 2003] that are grounded in parameterizations of the type used by O'Neill and Miller [1985]. Further analysis of the controlling mechanical conditions following the procedure developed here can be used to interpret phenomenological rules for the ice-sediment interactions in these systems in terms of fundamental physical quantities that can be measured. Turning this around, one might hope to use observations of freezing behavior in nature to infer the physical properties and environmental forcings that have produced periglacial landforms.

[48] The crude approximations used to obtain lens spacings and thicknesses in section 3.3 should be improved 
upon. The effects of thermal inertia, particularly at the onset of lens growth are expected to produce deviations in the precise values of $\lambda_{l n}, \lambda_{f n}, \lambda_{l m}$ and $\lambda_{f m}$ from those reported in Table 2. A more rigorous treatment of this dual movingboundary problem is in progress.

[49] At the onset of freezing, I have assumed that the first lens forms at a temperature such that the mechanical condition embodied in equation (21) is satisfied. It is important to recognize that this is an idealization. Implicit in any continuum description of porous media behavior is the assumption that average properties can be meaningfully defined over a small volume that contains a representative distribution of pores and particles. Clearly this is not the case at the initial time for the current problem. One might choose to instead regard the initial lens position as being controlled by the soil microstructure, for example, beginning once the freezing front extends down a particle diameter to the centroidal location of the first pores. Another alternative would be to postulate that freezing begins immediately at the ground surface with an initial lens temperature equal to the imposed surface temperature. Indeed, when macroscopic ponding occurs at the ground surface, the temperature at the base of the first lens should be largely controlled by heat flow through the puddle depth. Having a preexisting lens, such as a frozen puddle, effectively removes the need for considering a maximum frost heave pressure; the regime boundaries shown in Figure 6 would be compromised since some slow rate of heave could occur no matter what the overburden pressure. In these circumstances, the potential for steady growth of a single lens or the growth of multiple lenses still remain, though the precise locations of these regime boundaries would be adjusted from those calculated here.

\section{Conclusions}

[50] By examining the force balance conditions over an ice lens, in equation (6) I have shown that the product of the lens growth rate with the thickness of the partially frozen fringe that lies beneath it is equal to $\left(\mathbf{V}_{l} \cdot \hat{\mathbf{z}}\right)\left(z_{f}-z_{l}\right)=$ $H\left(T_{l}\right) \psi\left(z_{l}, T_{l}\right)$. The first of these functions $H\left(T_{l}\right)$ can be thought of as measuring the capacity of the lens to draw water through the underlying soil to cause heave; it is defined in terms of measurable characteristics of the porous medium, material properties, and the overburden (see, e.g., Figure 4). All dependence on the temperature profile is contained within the second function $\psi\left(z_{l}, T_{l}\right)$, which reflects deviations from a linear temperature gradient within the partially frozen fringe (e.g., see Figure 9). Having determined the mechanical conditions that dictate how lenses grow, an examination of the force balance conditions in the partially frozen fringe beneath the lens leads to equation (8) as a description of the location and temperature at which the effective stress borne by particle contacts vanishes and a new lens can grow (e.g., $P_{p}=0$ ). This introduces the concept of a critical heaving capacity $H_{c}\left(T, T_{l}\right)$, which is a second function of temperature, overburden, material properties, and measurable pore space characteristics that is defined to equal $H\left(T_{l}\right)$ at the point where a new lens is initiated when the temperature gradient in the fringe is linear (see, e.g., Figure 3). When the temperature gradient is not linear, the lens initiation condition generalizes to $H\left(T_{l}\right) \psi\left(z_{l}, T_{l}\right)=H_{c}\left(T, T_{l}\right) \psi(z, T)$, which accounts for temperature-dependent changes to the hydraulic resistance at different levels within the fringe.

[51] To illustrate how these mechanical conditions produce different regimes of freezing behavior during transient solidification, I analyzed the step-freezing problem in a uniform porous medium. This allowed us to identify regimes in which lenses can $\left(P_{0}<P_{\max }\right)$ or cannot $\left(P_{0}>\right.$ $\left.P_{\max }\right)$ be initiated, depending on the size of the overburden $P_{0}$ in comparison with the maximum frost heave pressure $P_{\max }$ defined by equation (22). Once the first lens forms, water begins to flow, and depending on whether the rate of fluid supply to the lens allows freezing and latent heat release that keeps pace with the rate of heat flow to the ground surface, the lens will either grow toward warmer temperatures or migrate toward colder temperatures. In the former case, the lens temperature adjusts until a stable steady state is reached and no further lens initiation occurs. In the latter case, the lens cools and the fringe thickens until eventually a new lens forms at a warmer temperature; subsequent freezing occurs along its contorted boundary until it cools sufficiently for yet another lens to form and the process repeats. These considerations led us to generate the regime diagrams in Figures 7 and 8 that delineate between the single lens, multiple lensing, and pore freezing regimes for three different model porous media. Approximations to the solution for the thermal problem described in equations (15)-(20). allow us to produce estimates for the lens thickness and spacing, as well as the accumulated total heave, as shown in Figure 11. I have discussed some of the ways in which this model can be modified and extended to consider several other geophysical phenomena beyond the simple one-dimensional freezing considered here. Efforts are in progress to apply similar methodologies to develop models that accurately homogenize the underlying microscopic interactions that produce the observed macroscopic behavior in several of these contexts.

\section{Appendix A: Formulae for Power Law Porous Media}

[52] The parameters listed in the lower third of Table 1 are chosen to represent the porous media solidification behavior in the form of the power law relations given in equations (9) and (10). In Table 2-6 of Andersland and Ladanyi [2004], ratios of water mass to dry mass of a range of porous media are reported in the form $w_{u}=A /\left(T_{m}-T\right)^{\beta}$, where $A$ is chosen to fit the empirical data when $T_{m}-T$ is measured in degrees centigrade. To convert to the form used in equation (10) I define $T_{f}$ so that $\left(T_{m}-T_{f}\right)^{\beta}=[(1-\phi) /$ $\phi]\left(\rho_{p} / \rho_{l}\right) A$, where the particle density is taken as $\rho_{p}=$ $2.65 \rho_{l}$. Figure C-14 of Andersland and Ladanyi [2004] contains a log-log plot of hydraulic conductivity $K$ as a function of $T_{m}-T$. Straight line fits to the data are used to obtain power law relations of the form used in equation (9), while noting that the permeability is defined so that $k=K \mu$ / $\left(\rho_{l}|\mathbf{g}|\right)$, where $\mathbf{g}$ is the acceleration of gravity. 
[53] Using the power law representations given in equations (9) and (10) I can write

$$
\begin{aligned}
& \int_{T}^{T_{f}} \phi S_{s} \mathrm{~d} T=\phi\left[T_{f}-T+\frac{T_{m}-T_{f}}{\beta-1}\left(\left(\frac{T_{m}-T_{f}}{T_{m}-T}\right)^{\beta-1}-1\right)\right], \\
& \int_{T}^{T_{f}} \frac{\left(1-\phi S_{s}\right)^{2}}{k} \mathrm{~d} T=\frac{T_{m}-T_{f}}{k_{0}}\left[\frac{(1-\phi)^{2}}{\alpha+1}\left(\left(\frac{T_{m}-T}{T_{m}-T_{f}}\right)^{\alpha+1}-1\right)\right. \\
& \quad+\frac{2(1-\phi) \phi}{\alpha-\beta+1}\left(\left(\frac{T_{m}-T}{T_{m}-T_{f}}\right)^{\alpha-\beta+1}-1\right) \\
& \left.+\frac{\phi^{2}}{\alpha-2 \beta+1}\left(\left(\frac{T_{m}-T}{T_{m}-T_{f}}\right)^{\alpha-2 \beta+1}-1\right)\right],
\end{aligned}
$$

where I assume that $\beta \neq 1, \alpha \neq-1, \alpha \neq \beta-1$ and $\alpha \neq$ $2 \beta-1$. These can be substituted into the expressions for $H\left(T_{l}\right)$ following (6) and $H_{c}\left(T, T_{l}\right)$ following equation (8). In the latter case, simple algebraic manipulation yields

$$
\begin{aligned}
H_{c}\left(T, T_{l}\right) & =\frac{\rho_{l} \mathcal{L} k_{0}}{\mu T_{m}}\left(\frac{T_{f}-T_{l}}{T_{m}-T_{f}}\right) \\
& \times \frac{\phi \frac{\beta}{\beta-1}\left(T_{m}-T_{f}\right)\left(1-\theta^{1-\beta}\right)-P_{0} T_{m} /(\rho \mathcal{L})}{\frac{(1-\phi)^{2}}{\alpha+1}\left(\theta^{\alpha+1}-1\right)+\frac{2(1-\phi) \phi}{\alpha-\beta+1}\left(\theta^{\alpha-\beta+1}-1\right)+\frac{\phi^{2}}{\alpha-2 \beta+1}\left(\theta^{\alpha-2 \beta+1}-1\right)},
\end{aligned}
$$

where $\theta \equiv\left(T_{m}-T\right) /\left(T_{m}-T_{f}\right)$. Noting that the first lens forms at the temperature that $H_{c}\left(T, T_{l}\right)=0$, I find that this occurs at

$$
T_{1 s t}=T_{m}-\left(T_{m}-T_{f}\right)\left(1-\frac{P_{0} T_{m}}{\rho \mathcal{L} \phi \beta} \frac{\beta-1}{T_{m}-T_{f}}\right)^{1 /(1-\beta)}
$$

For this reason, as indicated by equation (22), I do not expect any lenses to form when the overburden exceeds

$$
P_{\max }\left(T_{s}\right)=\frac{\rho \mathcal{L} \phi \beta}{T_{m}} \frac{T_{m}-T_{f}}{\beta-1}\left[1-\left(\frac{T_{m}-T_{f}}{T_{m}-T_{s}}\right)^{\beta-1}\right] .
$$

[54] Acknowledgments. This paper has benefitted greatly as a result of the careful comments made by the editor, Bob Anderson, and associate editor, Frederick Nelson, and the reviews by Andrew Fowler and Rorik Peterson. I also thank John Wettlaufer and Grae Worster for many stimulating discussions and especially their comments on an earlier version of this work. Funding was provided by NSF grant OPP0440841.

\section{References}

Abramowitz, M., and I. A. Stegun (1964), Handbook of Mathematical Functions, U.S. Gov. Print. Off., Washington, D. C.

Andersland, O. B., and B. Ladanyi (2004), An Introduction to Frozen Ground Engineering, 363 pp., CRC Press, Boca Raton, Fla.

Batchelor, G. K. (1967), An Introduction to Fluid Dynamics, Cambridge Univ. Press, New York.

Cahn, J. W., J. G. Dash, and H.-Y. Fu (1992), Theory of ice premelting in monosized powders, J. Cryst. Growth, 123, 101-108.

Carsey, F., A. Behar, A. L. Lane, V. Realmuto, and H. Engelhardt (2002), A borehole camera system for imaging the deep interior of ice sheets, J. Glaciol., 48, 622-628.

Christoffersen, P., and S. Tulaczyk (2003), Response of subglacial sediments to basal freeze-on: 1 . Theory and comparison to observations beneath the West Antarctic Ice Sheet, J. Geophys. Res., 108(B4), 2222, doi:10.1029/2002JB001935.

Clarke, G. K. C. (1987), Subglacial till-A physical framework for its properties and processes, J. Geophys. Res., 92, 9023-9036.

Dash, J. G. (1989), Thermomolecular pressure in surface meltingMotivation for frost heave, Science, 246, 1591-1593.
Dash, J. G., H. Y. Fu, and J. S. Wettlaufer (1995), The premelting of ice and its environmental consequences, Rep. Prog. Phys., 58, 115-167.

Dash, J. G., A. W. Rempel, and J. S. Wettlaufer (2006), The physics of premelted ice and its geophysical consequences, Rev. Mod. Phys., 78(3), $695-741$.

Fowler, A. C. (2003), On the rheology of till, Ann. Glaciol., 37, 55-59.

Fowler, A. C., and W. B. Krantz (1994), A generalized secondary frost heave model, SIAM J. Appl. Math., 54, 1650-1675.

Gilpin, R. R. (1980), A model for the prediction of ice lensing and frost heave in soils, Water Resour. Res., 16, 918-930.

Hales, T. C., and J. R. Roering (2005), Climate-controlled variations in scree production, Southern Alps, New Zealand, Geology, 33, 701704

Hallet, B., J. S. Walder, and C. W. Stubbs (1991), Weathering by segregation ice growth in microcracks at sustained sub-zero temperatures: Verification from an experimental study using acoustic emissions, Permafrost Periglacial Processes, 2, 283-300.

Horiguchi, K., and R. D. Miller (1983), Hydraulic conductivity functions of frozen materials, in Proceedings of the 4th 4th International Conference on Permafrost, pp. 504-508, Natl. Acad. Press, Washington, D. C.

Kessler, M. A., and B. T. Werner (2003), Self-organization of sorted patterned ground, Science, 299, 380-383.

Konrad, J.-M., and N. R. Morgenstern (1981), The segregation potential of a freezing soil, Can. Geotech. J., 18, 482-491.

Matsuoka, N. (2001), Direct observation of frost wedging in alpine bedrock, Earth Surf. Processes Landforms, 26, 601-614.

Miller, R. D. (1978), Frost heaving in non-colloidal soils, paper presented at 3rd International Conference on Permafrost, Natl. Res. Counc., Ottawa.

Murton, J. B., R. Peterson, and J.-C. Ozouf (2006), Bedrock fracture by ice segregation in cold regions, Science, 314, 1127-1129.

Nixon, J. F. D. (1991), Discrete ice lens theory for frost heave in soils, Can. Geotech. J., 28, 843-859.

O’Neill, K., and R. D. Miller (1985), Exploration of a rigid ice model of frost heave, Water Resour. Res., 21, 281-296.

Oxtoby, D. W. (1999), Nucleation and surface melting of ice, in Ice Physics and the Natural Environment, NATO ASI Ser, vol. 156, edited by J. S. Wettlaufer, J. G. Dash, and N. Untersteiner, pp. 23-38, Springer, New York.

Peterson, R. A., and W. B. Krantz (2003), A mechanism for differential frost heave and its implications for patterned-ground formation, J. Glaciol., 48, 69-80.

Plug, L. J., and B. T. Werner (2001), Fracture networks in frozen ground, J. Geophys. Res., 106, 8599-8613.

Plug, L. J., and B. T. Werner (2002), Nonlinear dynamics of ice-wedge networks and resulting sensitivity to severe cooling events, Nature, 417, 929-933.

Rempel, A. W., J. S. Wettlaufer, and M. G. Worster (2001), Interfacial premelting and the thermomolecular force: Thermodynamic buoyancy, Phys. Rev. Lett., 87, 088501.

Rempel, A. W., J. S. Wettlaufer, and M. G. Worster (2004), Premelting dynamics in a continuum model of frost heave, J. Fluid Mech., 498, $226-244$

Sletten, R. S., B. Hallet, and R. C. Fletcher (2003), Resurfacing time of terrestrial surfaces by the formation and maturation of polygonal patterned ground, J. Geophys. Res., 108(E4), 8044, doi:10.1029/ 2002JE001914.

Smith, M. W. (1985), Observations of soil freezing and frost heave in Inuvik, Northwest Territories, Canada, Can. J. Earth Sci., 22, $283-$ 290.

Smith, M. W., and A. R. Tice (1988), Measurement of unfrozen water content in soils, in 5th International Conference on Permafrost, edited by K. Sennest, pp. 473-477, Tapir, Trondhiem, Norway.

Swainson, I. P., and E. M. Schulson (2001), A neutron diffraction study of ice and water within a hardened cement paste during freeze-thaw, Cement Concrete Res., 31, 1821-1830.

Taber, S. (1930), The mechanics of frost heaving, J. Geol., 38, $303-$ 317

Tice, A. R., Y. Zhu, and J. L. Oliphant (1984), The effects of soluble salts on the unfrozen water contents of the Lanzhou, P.R.C., silt, Rep. 84-16, Cold Reg. Res. and Eng. Lab., Hanover, N. H.

Tulaczyk, S., W. B. Kamb, and H. F. Engelhardt (2000), Basal mechanics of Ice Stream B, West Antarctica: 1. Till mechanics, J. Geophys. Res., 105, $463-481$.

Walder, J., and B. Hallet (1985), A theoretical model of the fracture of rock during freezing, Geol. Soc. Am. Bull., 96, 336-346.

Walder, J., and B. Hallet (1986), The physical basis of frost weatheringToward a more fundamental and unified perspective, J. Arct. Alp. Res., $18,27-32$ 
Wettlaufer, J. S. (2001), The Stefan problem: Polar exploration and the mathematics of moving boundaries, in Die Zentralanstalt für Meteorologie und Geodynamik, 1851-2001, 150 Jahre Meteorologie und Geophysik in Osterreich, edited by C. Hammerl et al., pp. 420-435, Leykam, Graz, Austria.
Worster, M. G., and J. S. Wettlaufer (1999), The fluid mechanics of premelted liquid films, in Fluid Dynamics at Interfaces, edited by W. Shyy and R. Narayanan, pp. 339-351, Cambridge Univ. Press, New York.

A. W. Rempel, Department of Geological Sciences, University of Oregon, Eugene, OR 97403-1272, USA. 